\title{
Galactic Cosmic-Ray Anisotropies: Voyager 1 in the Local Interstellar Medium
}

\author{
J. S. Rankin ${ }^{1,2}$ (1) E. C. Stone ${ }^{2}$, A. C. Cummings ${ }^{2}$ (1) D. J. McComas ${ }^{1}(1)$, N. Lal ${ }^{3}$, and B. C. Heikkila ${ }^{3}$ \\ ${ }^{1}$ Department of Astrophysical Sciences, Princeton University, Princeton, NJ, USA \\ ${ }^{2}$ California Institute of Technology, Pasadena, CA, USA \\ ${ }^{3}$ Goddard Space Flight Center, Greenbelt, MD, USA \\ Received 2018 December 4; revised 2019 January 22; accepted 2019 January 31; published 2019 March 4
}

\begin{abstract}
Since crossing the heliopause on 2012 August 25, Voyager 1 observed reductions in galactic cosmic ray count rates caused by a time-varying depletion of particles with pitch angles near $90^{\circ}$, while intensities of particles with other pitch angles remain unchanged. Between late 2012 and mid-2017, three large-scale events occurred, lasting from $\sim 100$ to $\sim 630$ days. Omnidirectional and directional high-energy data from Voyager 1's Cosmic Ray Subsystem are used to report cosmic ray intensity variations. Omnidirectional ( $\gtrsim 20 \mathrm{MeV})$ proton-dominated measurements show up to a $3.8 \%$ intensity reduction. Bidirectional $(\gtrsim 70 \mathrm{MeV})$ proton-dominated measurements taken from various spacecraft orientations provide insight about the depletion region's spatial properties. We characterize the anisotropy as a "notch" in an otherwise uniform pitch angle distribution of varying depth and width centered about $90^{\circ}$ in pitch angle space. The notch averages $22^{\circ}$ wide and $15 \%$ deep, signifying a depletion region that is broad and shallow. There are indications that the anisotropy is formed by a combination of magnetic trapping and cooling downstream of solar-induced transient disturbances in a region that is also likely influenced by the highly compressed fields near the heliopause.
\end{abstract}

Key words: astroparticle physics - cosmic rays - ISM: magnetic fields - scattering - shock waves - turbulence

\section{Introduction}

Voyager 1's crossing of the heliopause on 2012 August 25, was marked by sharp increases in low-energy galactic cosmic rays (GCRs) and corresponding sudden decreases in anomalous cosmic rays, as observed by the Cosmic Ray Subsystem (CRS) and Low Energy Charged Particle (LECP) instruments (Krimigis et al. 2013; Stone et al. 2013; Webber \& McDonald 2013). In the wake of Voyager's interstellar arrival, LECP observed an unexpected anisotropy in the GCRs characterized by a clear reduction in $>211 \mathrm{MeV}$ proton intensities for particles entering their bidirectional telescope when viewing perpendicular to the magnetic field. Several extended, timedependent events have continued to occur during Voyager l's interstellar journey beyond the heliopause.

In addition to the GCR anisotropies, Voyager l's four working instruments observed several signatures of transient disturbances in the interstellar medium. Burlaga et al. (2013) and Burlaga \& Ness (2016) reported several weak, laminar, quasiperpendicular, subcritical, resistive disturbances observed by the magnetometer. Gurnett et al. (2013, 2015) detail a series of locally generated electron plasma emissions detected by the Plasma Wave Subsystem (PWS) instrument. Moreover, they compare the PWS-measured events with GCR disturbances seen by CRS and LECP and describe their relationship as analogous to precursor effects often observed in the "foreshock" region upstream of planetary bow shocks.

Evidence suggests that the transient events and GCR anisotropies may be related. For example, Jokipii \& Kóta (2014) and Kóta \& Jokipii (2017) showed numerical simulations indicating that a gradual compression, followed by a slow weakening of the magnetic field, may account for the pitch angle and time profiles of both transient GCR increases and anisotropic decreases. These authors interpreted the pitch angle anisotropies to arise from particle trapping and adiabatic cooling behind these weak disturbances.

While Voyager 1 was making these detailed observations of the particle distributions just beyond the heliopause, the
Interstellar Boundary Explorer (IBEX, McComas et al. 2009a) was imaging the 3D properties and structure of the heliosphere's global interaction with the interstellar medium. In particular, IBEX discovered a "ribbon" of enhanced energetic neutral atom (ENA) emissions associated with the local interstellar magnetic field, which drapes around the heliosphere (McComas et al. 2009b; Schwadron et al. 2009). The ribbon provides the best determination of the external field direction (Funsten et al. 2009 , 2013) and magnitude ( $0.29 \mathrm{nT})$ at great distances (>1000 au) (Zirnstein et al. 2016). The draping and compression of this interstellar field around the outside of the heliopause leads to higher magnetic field strengths at Voyager 1, consistent with its local observations (Pogorelov et al. 2017) and even higher field strengths closer to the heliopause in the IBEX ribbon directions (McComas et al. 2009a; Pogorelov et al. 2011).

IBEX observations also revealed the importance of the interstellar magnetic field in shaping the global heliosphere. These showed that the interstellar medium's magnetic pressure produces large-scale asymmetries in the heliosphere's global structure, with the largest compression and greatest pressure region in the inner heliosheath, between the termination shock and heliopause, $\sim 20^{\circ}$ south and slightly offset toward the port side from the interstellar upwind direction (Schwadron et al. 2014). This offset pressure maximum causes asymmetric plasma flows in the inner heliosheath and naturally explains the unexpected flow directions observed by Voyager 2 (McComas \& Schwadron 2014). IBEX observations and the global asymmetries they expose in the heliopause's shape are also important for understanding the detailed particle distributions observed by Voyager 1, as we show in this study.

In this article, we focus on CRS measurements of the GCR anisotropy, presenting additional information about these unusual events through measurements of proton-dominated intensities. We describe CRS telescope modes that are relevant to viewing the anisotropy and report observations for varying spacecraft orientations in Section 2. In Section 3, we model the temporal and spatial behavior of the unexpected pitch angle 


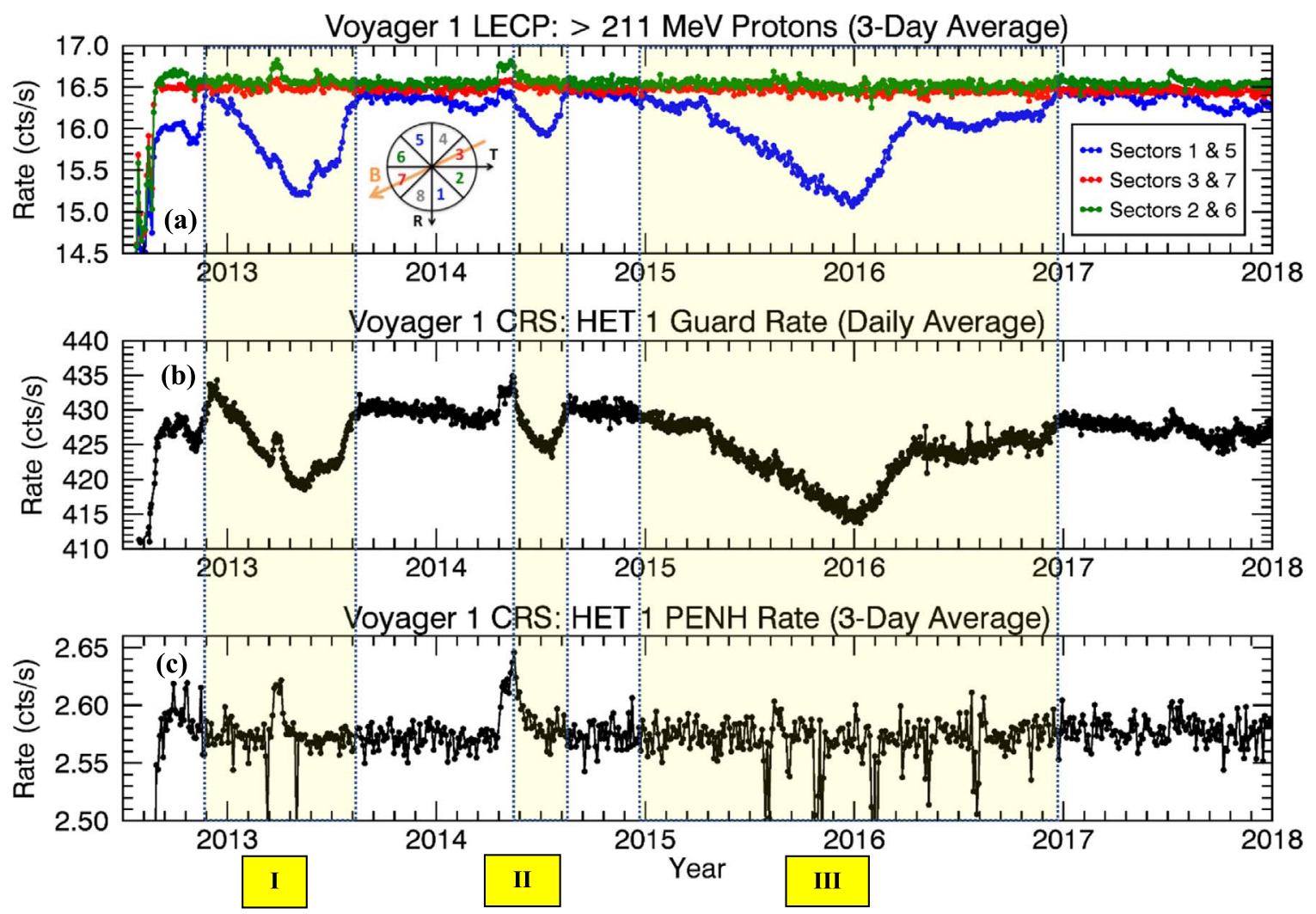

Figure 1. LECP and CRS counting rates in the LISM from 2012.5 through 2017. The three largest anisotropy episodes (shaded in yellow) last $\sim 265$ (I), $\sim 100$ (II), and $\sim 630$ (III) days, respectively. (a) LECP's $>211 \mathrm{MeV}$ protons reveal the GCR pitch angle anisotropy. The magnetic field direction lies in Sectors 3 and 7 , while Sectors 1 and 5 are approximately perpendicular to the field direction, as illustrated by the circular diagram (background-corrected data is courtesy of Rob Decker and the LECP team; for LECP's noncorrected, publicly available data, see http://sd-www.jhuapl.edu/VOYAGER/index.html). (b) CRS's omnidirectional guard rate ( $20 \mathrm{MeV}$; proton-dominated) from anticoincidence counters on the HET 1 telescope shows time dependence similar to that of LECP's Sectors 1 and 5. (c) CRS's bidirectional PENH rate on HET 1 ( $\gtrsim 70 \mathrm{MeV}$; proton-dominated) is fairly steady in the LISM, in agreement with LECP's bidirectional rates in Sectors 2 and 6 and 3 and 7. Two types of deviation arise from (1) shock-related increases (e.g., 2014.35) and (2) decreases observed during 70 ${ }^{\circ}$-offset spacecraft maneuvers (e.g., 2015.59).

phenomena, and in Section 4, we incorporate the results into three types of simulated response functions for comparison with observations. Finally, in Section 5, we explore magnetic trapping and shock-related adiabatic cooling as possible physical mechanisms for producing the anisotropy.

\section{Particle Anisotropy Observations}

CRS's double-ended high-energy telescopes (HETs 1 and 2) have geometry factors and energy ranges appropriate for observing GCR intensities and spectra in the local interstellar medium (LISM). Each telescope is composed of circular energetic particle detectors arranged in a cylindrical stack. Both HETs consist of seven silicon solid-state detectors $(\mathrm{C} 1$ through $\mathrm{C} 4)$ with annular guard rings $(\mathrm{G})$ that operate as omnidirectional anticoincidence counters. The end detectors consist of two thin detectors on the A-end (A1 and A2) and two curved detectors on the B-end (B1 and B2) (Stone et al. 1977). To provide directional measurements for multiple species over various energy ranges, CRS telescopes operate in multiple coincidence modes. Those of relevance to this study include HET 1 and 2's bidirectional penetrating mode (PENH; proton-dominated, $\left.{ }^{4} \gtrsim 70 \mathrm{MeV}\right)$ and omnidirectional mode (Guards; proton-dominated, $\gtrsim 20 \mathrm{MeV}$ ).

Figure 1 shows LECP and CRS count rates in the LISM from 2012.5 through 2017. LECP's > $211 \mathrm{MeV}$ protons show

\footnotetext{
4 In addition to protons, PENH is $\sim 25 \%$ electrons and $\sim 5 \%$ heavier nuclei ( $\gtrsim 70 \mathrm{MeV} /$ nuc). See Cummings et al. (2016) for more details on the constituents of CRS rates.
}

the anisotropy's signatures in the form of long-duration, timevarying intensity changes, present in Sectors 1 and 5 but not in other sectors. LECP has an advantage for viewing the pitch angle anisotropy because its stepper-motor platform routinely steps through eight viewing directions, as indicated by the circular diagram in Figure 1(a).

CRS's omnidirectional counters (Figure 1(b)) continuously monitor the temporal intensity changes without providing pitch angle information. Nevertheless, the omnidirectional guard rates have the highest statistics of all the rates available on CRS (several hundred counts $\mathrm{s}^{-1}$ ) and show a time-varying intensity response similar to LECP's. Detecting the anisotropy using directional observations presents a greater challenge. CRS's telescopes are body-fixed on the three-axis stabilized spacecraft, and the HET 1 and HET 2 fields of view do not typically observe particles with $\sim 90^{\circ}$ pitch angles, so their nominal rates are not sensitive to the anisotropy (see for example, HET 1's bidirectional PENH rate in Figure 1(c)). However, data taken during occasional spacecraft maneuvers provide an opportunity to examine the pitch angle variation of the intensity at specific times.

\subsection{Magnetometer Roll Maneuvers and Observations}

Magnetometer roll maneuvers are performed approximately six times per year for magnetometer calibration purposes. They originally consisted of 10 successive $360^{\circ}$ turns about the spacecraft's Earth-pointing axis (approximately $\hat{R}$ in the $R, T, N$ 

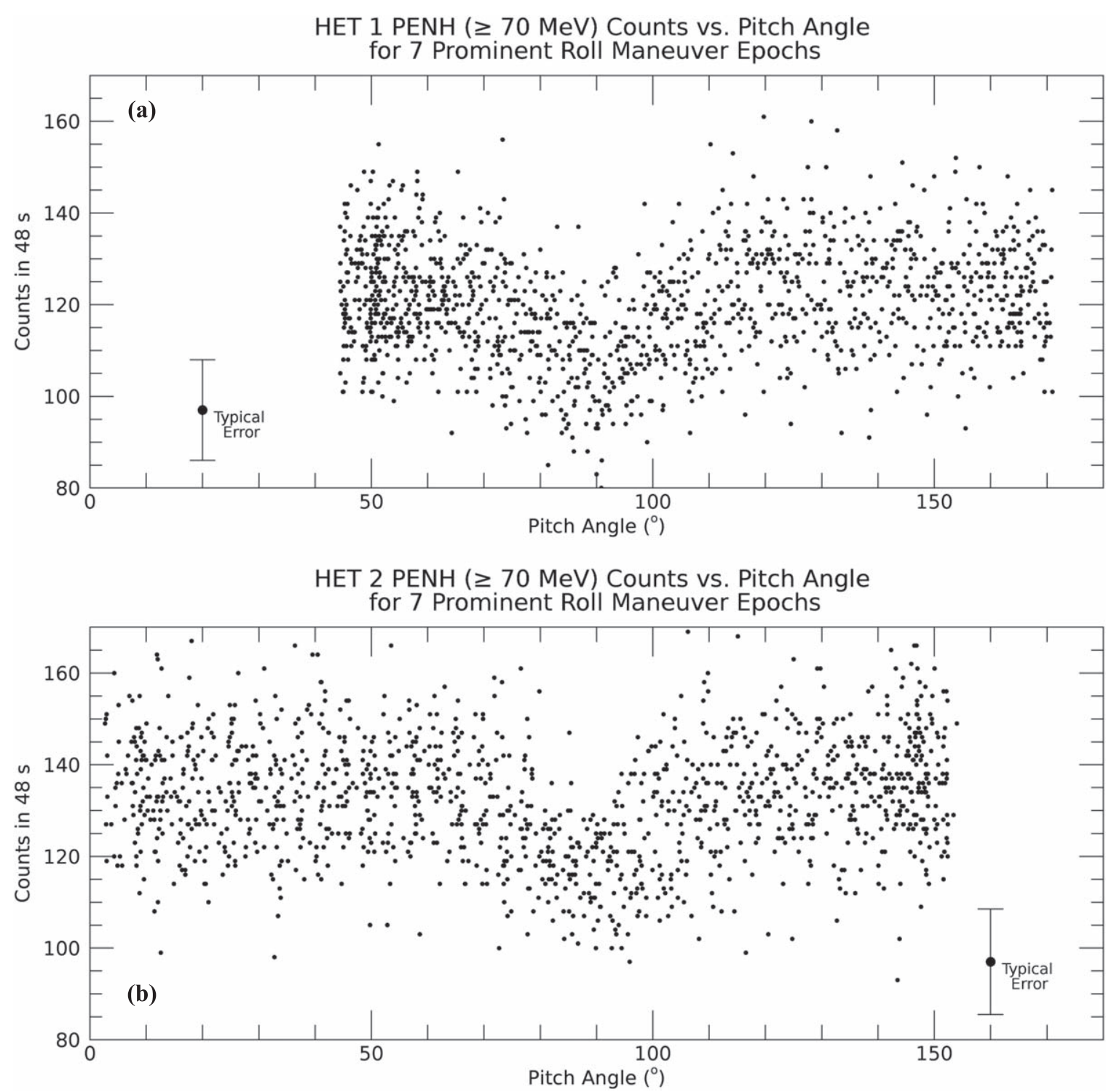

Figure 2. Superposition of seven prominent HET 1 (a) and HET 2 (b) roll maneuver intervals of varying anisotropy magnitudes arranged in pitch angle space (see Appendix A, Tables 3 and 4 for selected intervals).

coordinate system), ${ }^{5}$ but as of 2017 they are performed with a reduced number of turns because of power limitations. During the 10-roll period $(\sim 5.6 \mathrm{hr})$, CRS telescope fields of view smoothly and continuously rotate $360^{\circ}$ every $2000 \mathrm{~s}\left(0^{\circ} .18 / \mathrm{s}\right)$, which translates to an $8^{\circ} .6$ angular averaging per point in the highest-resolution data (48 s). "Clock angle" refers to the angle of the boresight in the $N-T$ plane with the $\hat{N}$-axis as the origin and the angle increasing toward $\hat{T}$. "Roll interval" refers to the set of 10 turns that took place on a particular day (e.g., the 2015-310 interval occurred on day 310 of 2015). Knowing the roll rate, the magnetic field direction, and the clock angle orientation of a telescope's boresight enables the average pitch angle of particles entering the telescope to be determined during each $48 \mathrm{~s}$ period throughout a roll maneuver.

HET 1 and HET 2 bidirectional PENH measurements during roll maneuvers confirm that the reduction observed by LECP's

\footnotetext{
$5 R, T, N$ is a spacecraft-centered coordinate system where $\hat{R}$ is the Sun-tospacecraft vector, $\hat{T}$ is the cross product of the Sun's rotation vector with $\hat{R}$, and $N$ completes the triad of the right-handed system.
}

Sectors 1 and 5 (Figure 1(a)) and CRS's omnidirectional rates (Figure 1(b)) results from a pitch angle anisotropy. Moreover, roll maneuver data provide the clearest measure of the anisotropy's spatial distribution. Figure 2 displays a superposition of HET 1 (Figure 2(a)) and HET 2 (Figure 2(b)) rates during seven rolls where the anisotropy is most prominent (selected intervals are indicated in Tables 3 and 4 of Appendix A). Although the effects of its time-variable magnitude are also present, not only does the anisotropy occur within a region that is perpendicular to the magnetic field - in agreement with LECP's observations-but it is distributed about $90^{\circ}$ pitch angle.

\section{2. $70^{\circ}$-offset Maneuvers and Observations}

$70^{\circ}$-offset maneuvers were introduced on Voyager 1 in 2011 March to provide a way for LECP to measure heliosheath plasma flow velocity in the direction not seen in its usual configuration (Decker et al. 2012) and were discontinued in 2017. Like magnetometer roll maneuvers, they require the spacecraft to roll about the $\hat{R}$-axis. However, rather than rolling continuously, the spacecraft rotates to a clock angle offset of $70^{\circ}$ and parks for up to 


\section{HET 1 PENH $70^{\circ}$-Offset Observations: \\ Manuever Sequence 2015, Days 296 through 312}
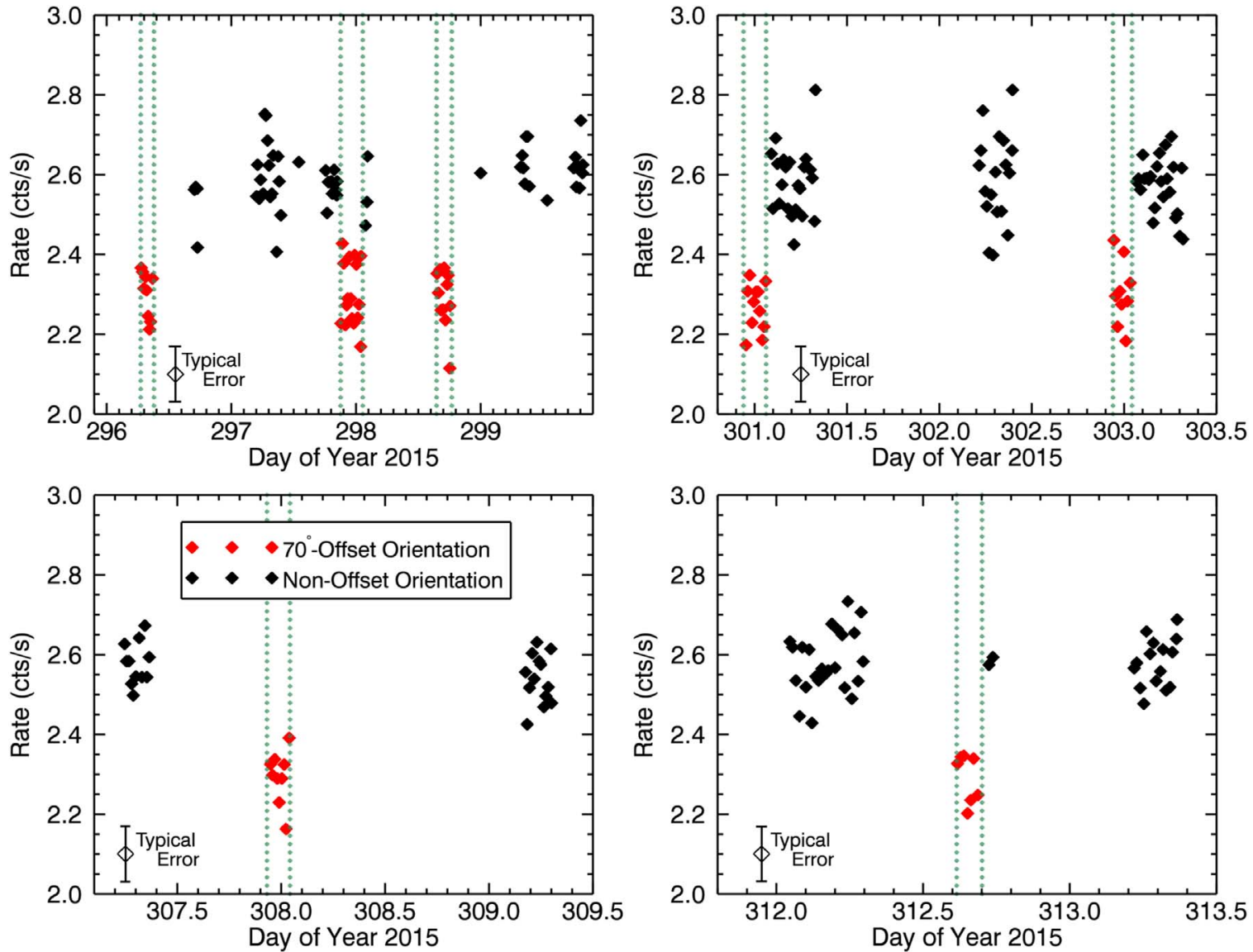

Figure 3. HET $1 \mathrm{PENH}$ ( $\gtrsim 70 \mathrm{MeV}$; proton-dominated) $70^{\circ}$-offset observations for a full sequence of maneuvers that took place in 2015 , on days 296 to 312 . Note that offset maneuvers take place within a subset of time over a period of multiple days, in contrast with roll maneuvers, which take place on a single day. The roll maneuver nearest to this 2015-296 offset occurred on day 310 of 2015. HET 1's average $70^{\circ}$-offset boresight pitch angle during this time is $79^{\circ} .3$ (A-end). Points are averaged over $\sim 480 \mathrm{~s}$ intervals for visualization purposes. The large data gaps show Voyager l's daily gaps in communication with Earth. The dotted lines denote the times when the spacecraft was fixed in the offset position. The red points mark observations taken while the spacecraft was parked at the $70^{\circ}$-clock-angle offset from its usual position. The black points represent values obtained while the spacecraft was in its nominal orientation. Because HET 1's $\sim 40^{\circ}$-wide field of view includes $90^{\circ}$ pitch angle during $70^{\circ}$-offsets (Table 1), it sees a reduction of counts indicative of the anisotropy.

Table 1

A Summary of CRS Telescope Boresight Directions in Pitch Angle Space

\begin{tabular}{lccc}
\hline \hline Telescope & Average Nominal Boresight Pitch Angle & Average $70^{\circ}$-offset Boresight Pitch Angle & Field of View (Full Angle) \\
\hline HET 1 & $136^{\circ} \pm 3^{\circ}$ (A-end) $44^{\circ} \pm 3^{\circ}$ (B-end) & $77^{\circ} \pm 3^{\circ}$ (A-end) $103^{\circ} \pm 3^{\circ}$ (B-end) & $40^{\circ}($ PENH) \\
HET 2 & $31^{\circ} \pm 4^{\circ}$ (A-end) $149^{\circ} \pm 4^{\circ}$ (B-end) & $69^{\circ} \pm 3^{\circ}$ (A-end) $111^{\circ} \pm 3^{\circ}$ (B-end) & $40^{\circ}$ (PENH)
\end{tabular}

Note. Particles entering a given telescope travel in directions opposite to the telescope's average boresight direction and field of view. Averages were determined using telescope and magnetic field directions from $\sim 2012.65$ to 2017.0. The average magnetic field during this period was $(0.143,-0.401,0.179) \mathrm{nT}$ in $R, T, N$. Uncertainties primarily reflect the small variations in the magnetic field direction.

5 hours before returning to its usual orientation. These maneuvers typically occur on consecutive days over a multiple-day period, usually near times of roll maneuvers.

For each offset period, we combine counts from multiple days and normalize to temporally adjacent non offset values to determine a relative intensity change arising from the pitch angle anisotropy $\left(\delta_{70^{\circ}}\right)$. Table 1 compares average HET 1 and 2 boresight pitch angles for times when the spacecraft is in its usual configuration and to those during $70^{\circ}$-offsets. In Figure 3 we show the average HET 1 PENH rates during the 2015-296 "offset interval," where DOY 296 is the first day of the sequence of seven maneuvers that took place on days 296 to 312 of 2015-this is the interval nearest to the 2015-310 roll maneuver. During $70^{\circ}$-offsets, HET 1's field of view overlaps significantly with $90^{\circ}$ pitch angle (Table 1 ), thus enabling these fixed-orientation measurements to complement roll maneuver and omnidirectional observations of the pitch angle anisotropy.

\subsection{Omnidirectional Observations}

We calculate the omnidirectional intensity reduction $\left(\delta_{\text {omni }}\right)$ by comparing observations of each period's daily average to 

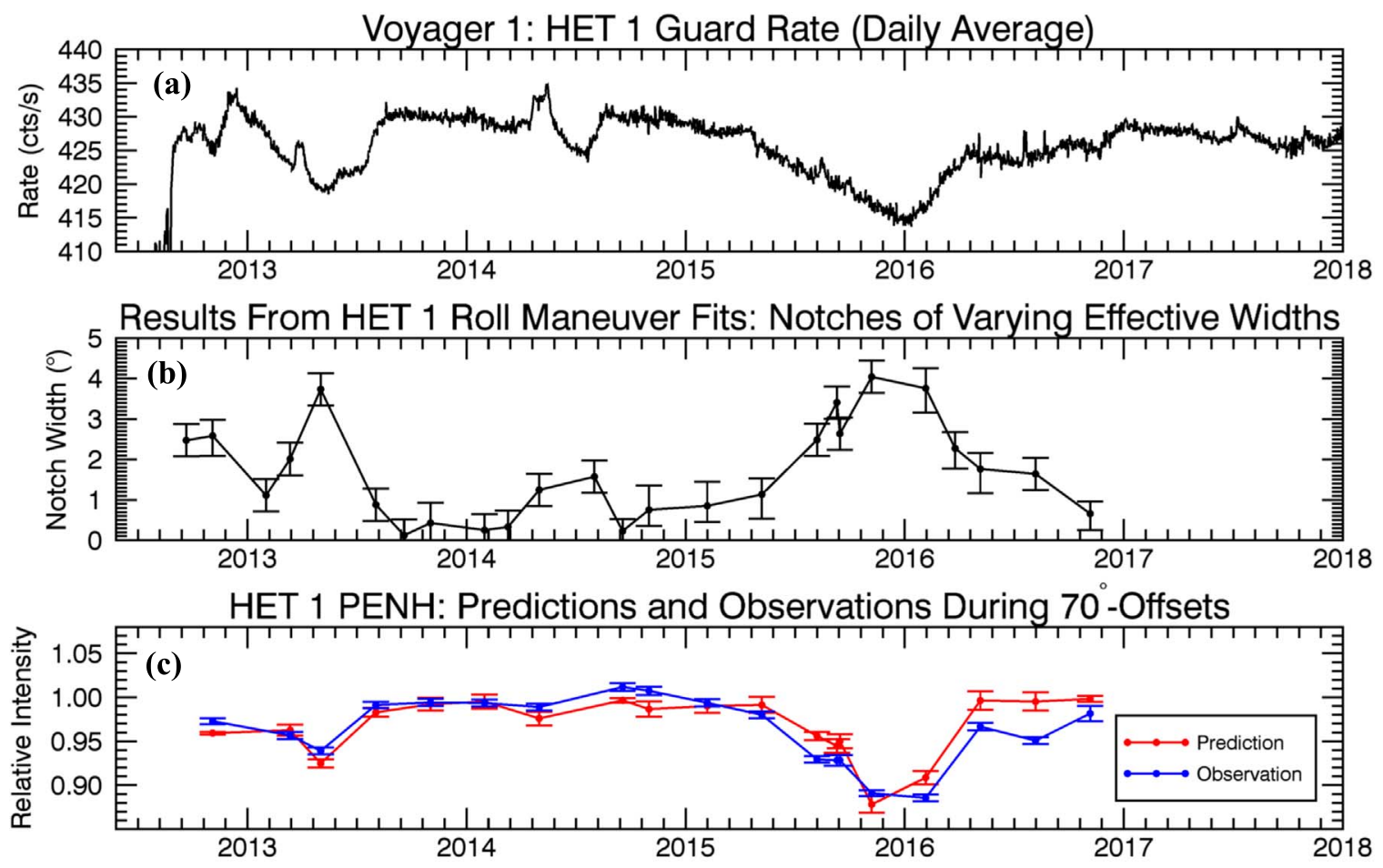

HET 1 Guard Rate: Predictions and Observations

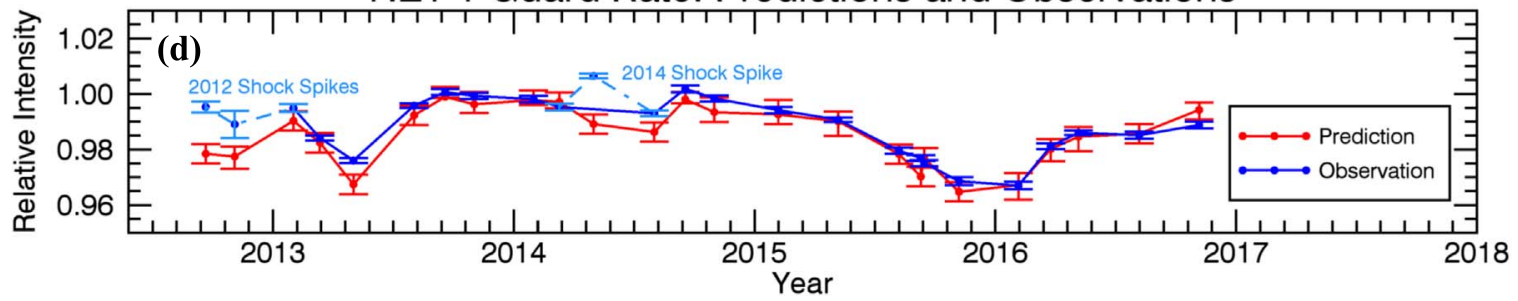

Figure 4. Results from Model \#1 simulations and comparison with observations: HET 1. (a) HET 1's omnidirectional guard rate ( $2020 \mathrm{MeV}$; proton-dominated) shows the time-varying GCR intensity reductions caused by the pitch angle anisotropy. (b) Effective notch widths from fits to HET 1's bidirectional PENH rate ( $70 \mathrm{MeV}$; proton-dominated) during 25 roll maneuvers from late 2012 through 2016 . We use these widths to generate the results shown in panels (c) and (d). (c) $70^{\circ}$ offset simulations and observations near the 25 roll intervals for HET 1's bidirectional PENH rate ( $>70 \mathrm{MeV}$; proton-dominated). Observed intensities are normalized to temporally adjacent non offset rates, while simulated intensities are normalized to modeled response function values without a notch. (d) Omnidirectional simulations and observations during the 25 roll intervals for HET 1's guard rate ( $\gtrsim 20 \mathrm{MeV}$; proton-dominated). Observed intensities are normalized to the average values during the 2013.6 to 2014.1 period when count rates are relatively uniform and isotropic, while simulated intensities are normalized to modeled response function values without a notch.

the average rate during 2013.6 to 2014.1 - a time when the pitch angle anisotropy is not prominent (note the steady rates in Figures 1(a) and (b)). The average isotropic rates used for normalization are $430.01 \pm 0.06$ counts $^{-1}$ for HET 1 and $382.38 \pm 0.06$ counts s $^{-1}$ for HET 2 .

The three main episodes of GCR intensity changes caused by the pitch angle anisotropy (Figure 1(b)) last on the order of 265 (region I), 100 (region II), and 630 (region III) days and exhibit at most $2.6 \%, 1.3 \%$, and $3.8 \%$ intensity reductions, respectively. A fundamental characteristic of the anisotropy that is supported by CRS's omnidirectional, roll maneuver, and $70^{\circ}$-offset observations is that these long-duration intensity changes arise primarily from the pitch angle anisotropy, as opposed to effects such as solar modulation, a radial gradient, or diffusive or convective flows.

\section{Characterizing the Anisotropy}

We model the anisotropy by generating particle distributions that are isotropic except for a "notch" centered at $90^{\circ}$ pitch angle. Such a notch of missing particles could be either partial or complete, so we base our simulation on two parameters-the notch's width and its depth-and compare the results to the overall reduction in the observed omnidirectional and directional GCR intensities. The actual pitch angle distribution might be more complicated than even a partially depleted, field-perpendicular notch, which would likely be difficult to resolve from the observations. This is because CRS's omnidirectional and directional rates are a mixture of temporal (48 s) and spatial averaging. In addition, the infrequency of spacecraft maneuvers ( $\sim 6$ times/year) adds to the statistical limitations of the directional data. Nonetheless, we argue that a missing notch in the pitch angle distribution is clearly the best first-order approximation to any more complicated distribution.

To obtain the results in the following section, we consider two approaches for setting limits on the notch's characteristics: (1) an empty notch (Model \#1: variable width, 100\% depth) and (2) a partially filled notch (Model \#2: variable width and depth). Appendices A and B describe the empty and partially filled notch models, respectively. In both cases, we define the model pitch angle distributions and determine the detailed instrument response to each type of distribution. 

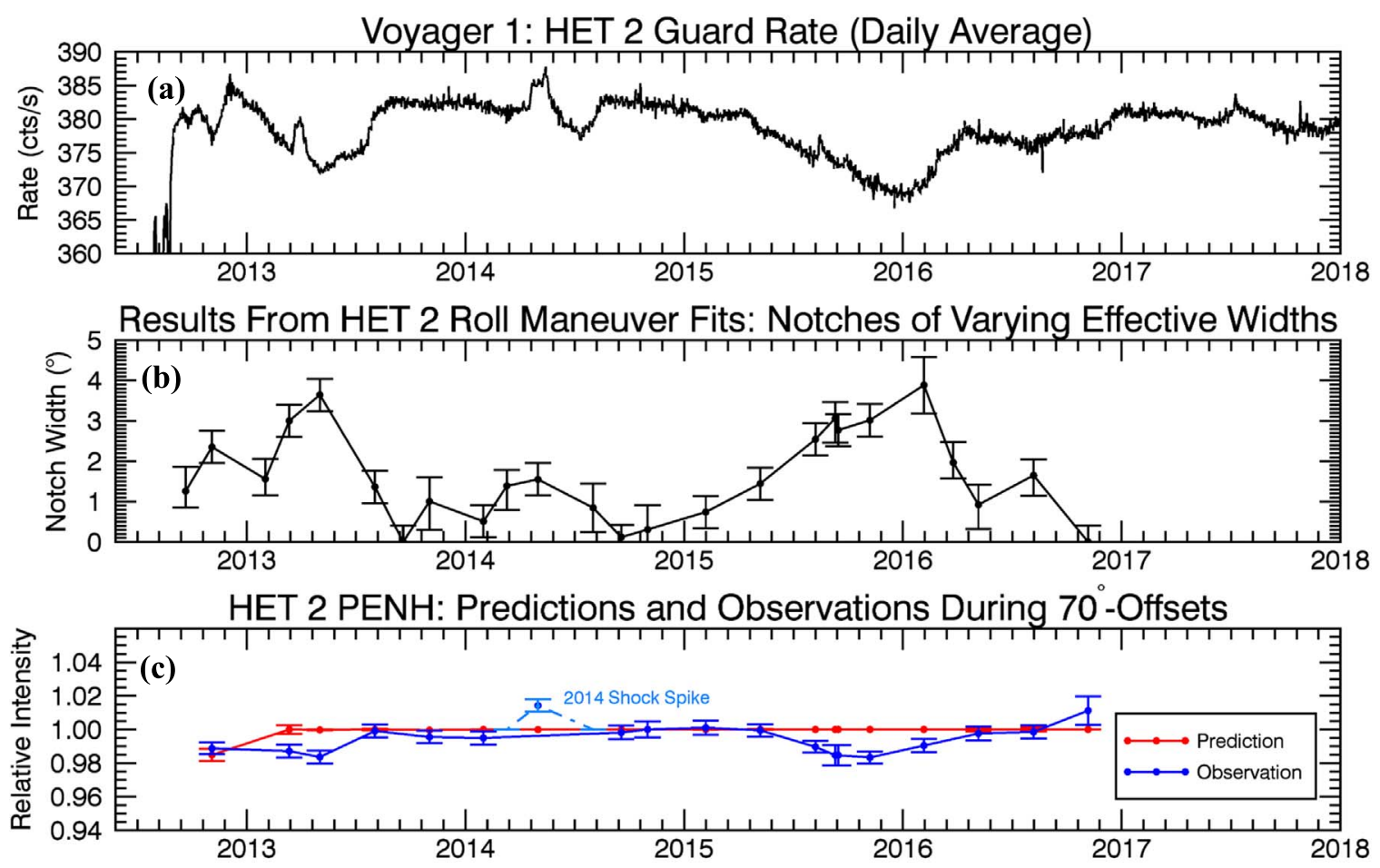

HET 2 Guard Rate: Predictions and Observations

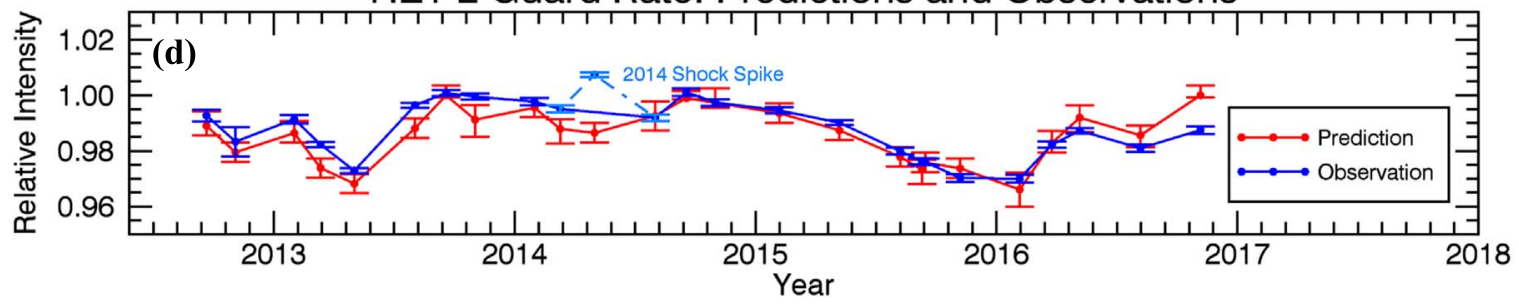

Figure 5. Results from Model \#1 simulations and comparison with observations for HET 2. (a) HET 2's omnidirectional guard rate ( $220 \mathrm{MeV}$; proton-dominated). (b) Effective notch widths from fits to HET 2's bidirectional PENH rate ( $270 \mathrm{MeV}$; proton-dominated) during 25 roll maneuvers from late 2012 through 2016. (c) $70^{\circ}$-offset simulations and observations near the 25 roll intervals for HET 2's bidirectional PENH rate ( $\gtrsim 70 \mathrm{MeV}$; proton-dominated). (d) Omnidirectional simulations and observations during the 25 roll intervals for HET 2's guard rate ( $220 \mathrm{MeV}$; proton-dominated).

\section{Results}

\subsection{Model \#1 Results and Comparison with Observations}

We show the results from Model \#1's best fits to HET 1's roll maneuver observations in Figure 4 (see also Table 3 of Appendix A). The data were taken during 25 maneuvers that occurred from late 2012 (shortly after the heliopause crossing) to the end of 2016, when the number of rolls per maneuver was reduced. The effective widths range from $0^{\circ}$ to $\sim 4^{\circ}$ (Figure 4(b)). Overall, this model agrees well with respective $70^{\circ}$-offset (Figure 4(c)) and omnidirectional (Figure 4(d)) observations for HET 1.

Similar results derived from the best fits to HET 2's roll maneuvers are shown in Figure 5 and listed in Table 4 of Appendix A. HET 2's widths also vary from $0^{\circ}$ to $\sim 4^{\circ}$ (Figure 5(b)) and results from Model \#1's simulated omnidirectional notch response function agree with observations (Figure 5(d)). However, the $70^{\circ}$-offset results are not consistent (Figure 5(c)) with the results of this model. According to our simulation, HET 2 should not observe an intensity change, yet it observes small, but still statistically significant anisotropic decreases.

Resolving this $70^{\circ}$-offset discrepancy requires a shift in boresight pitch angle of $\sim 8^{\circ}$, which theoretically might be explained by considering uncertainties in the telescope's assumed pointing direction and the measured magnetic field direction. However, the adjustment needed is too large to be attributed to uncertainty in telescope orientation and is also beyond the range of the magnetometer's uncertainties. An added complication is that changing the magnetic field direction also affects the results for HET 1.

Instead, the most likely way to resolve HET 2's inconsistencies is by allowing for a wider notch. However, we cannot achieve this without generating inconsistencies in HET 1 if we maintain Model \#1's $100 \%$ depth assumption. For example, HET 2 's observations $(1.7 \% \pm 0.4 \%)$ during the 2015-296 offset interval (2015-310 roll interval) can be reproduced by an effective notch width of $19^{\circ} .1 \pm 0^{\circ} .8$. Yet, this same width applied to HET 1 yields a simulated $56.2 \% \pm 2.2 \%$ relative intensity reduction compared to its $11.0 \% \pm 0.3 \%$ observation. Thus, we resolve these issues by introducing a variable depth parameter through Model \#2's broader, partially filled notch.

\subsection{Model \#2 Results and Comparison with Observations}

We report notch widths and depths from Model \#2's best fits to HET 1 and HET 2 roll maneuver observations in 

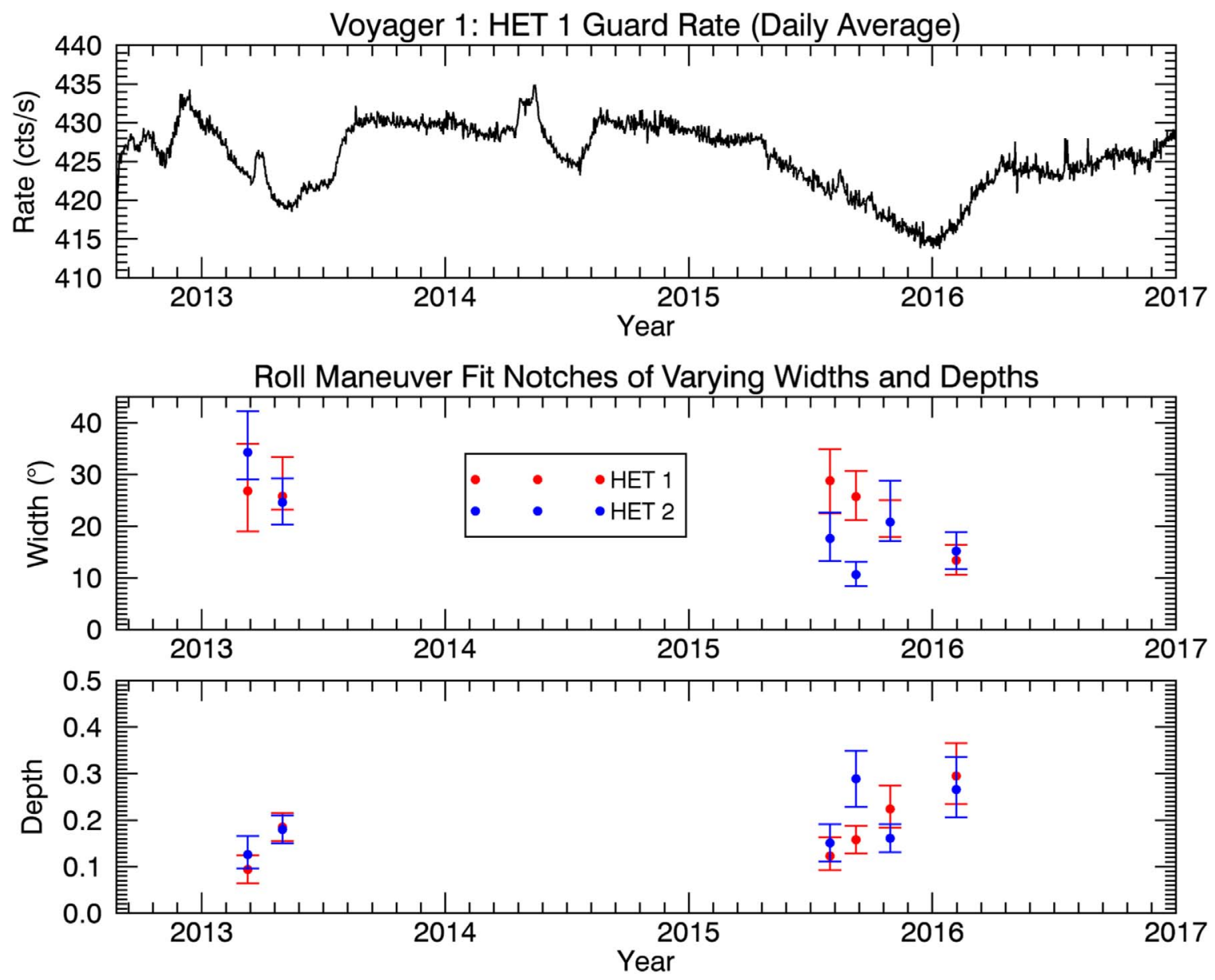

Figure 6. Widths and depths predicted from HET 1 (red) and HET 2 (blue) roll maneuver fits for notches of varying widths and depths. Error bars denote $1 \sigma$ uncertainties. We note that the depths signify the relative intensity deviation from isotropy; for example, a depth of 0.1 means that the intensity within the notch is reduced by $10 \%$ compared to the isotropic baseline (see Appendix B for more details).

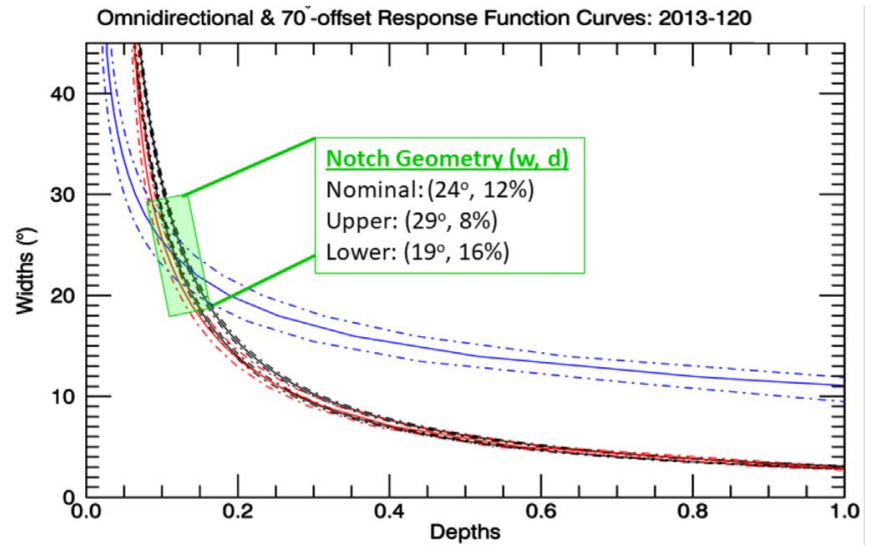

Figure 7. Superposition of HET 1 and HET 2 omnidirectional (black dashed) and $70^{\circ}$-offset response function curves (HET 1 in red, HET 2 in blue) for the 2013-120 offset interval. Note that agreement is achieved by a broad, shallow notch as opposed to a narrow, deep notch (e.g., at the limit of Model \#1).

Figure 6, focusing on the six intervals where the anisotropy is most prominent (see Figure 5(b) and Appendix B, Tables 7 and 8)

HET 1 and 2 results agree for five of the six intervals. HET 2's 2015-252 offset $(\sim 2015.69)$ had a poor fit with a $P$-value of $0.50 \%$ (Table 8, Appendix B). Perhaps a small shock enhancement that occurred near 2015-224 or plasma oscillations that began on 2015-247 contributed to this outlier.

As an independent verification of the notch's parameters, we superimpose simulated HET 1 and $270^{\circ}$-offset and omnidirectional response function curves to constrain widths and depths (detailed in Appendix B). To illustrate, Figure 7 displays a superposition of HET 1 and 2 omnidirectional and $70^{\circ}$-offset response function curves for the 2013-120 offset. In general, HET 1 and 2 agree for shallow, broad notches (e.g., around $24^{\circ}$ wide and $12 \%$ deep) as opposed to narrow, deep notches (e.g., toward Model \#1's 100\% depth limit).

A complication of this approach is that HET 1's omnidirectional and $70^{\circ}$-offset curves do not always intersect because of the combination of uncertainties in the reported $B$-field and the particular sensitivity of HET 1's response function to pitch angle. To resolve the discrepancy for the affected offset intervals, we select field values that fall within the magnetometer's uncertainties by minimizing each component's deviation from reported observations (see Appendix B, Section B. 2 for further details). Figure 8 shows the resulting notch geometries.

For four intervals, HET 1's $70^{\circ}$-offset curves matched the omnidirectional curves over a broad range of widths and depths, and therefore could not effectively constrain the notch's geometry. However, for two intervals (2015-208 and 2016-31), they were sufficiently different to allow HET 1 to confirm the broad, shallow notch seen by HET 2 . Regarding uncertainties, 

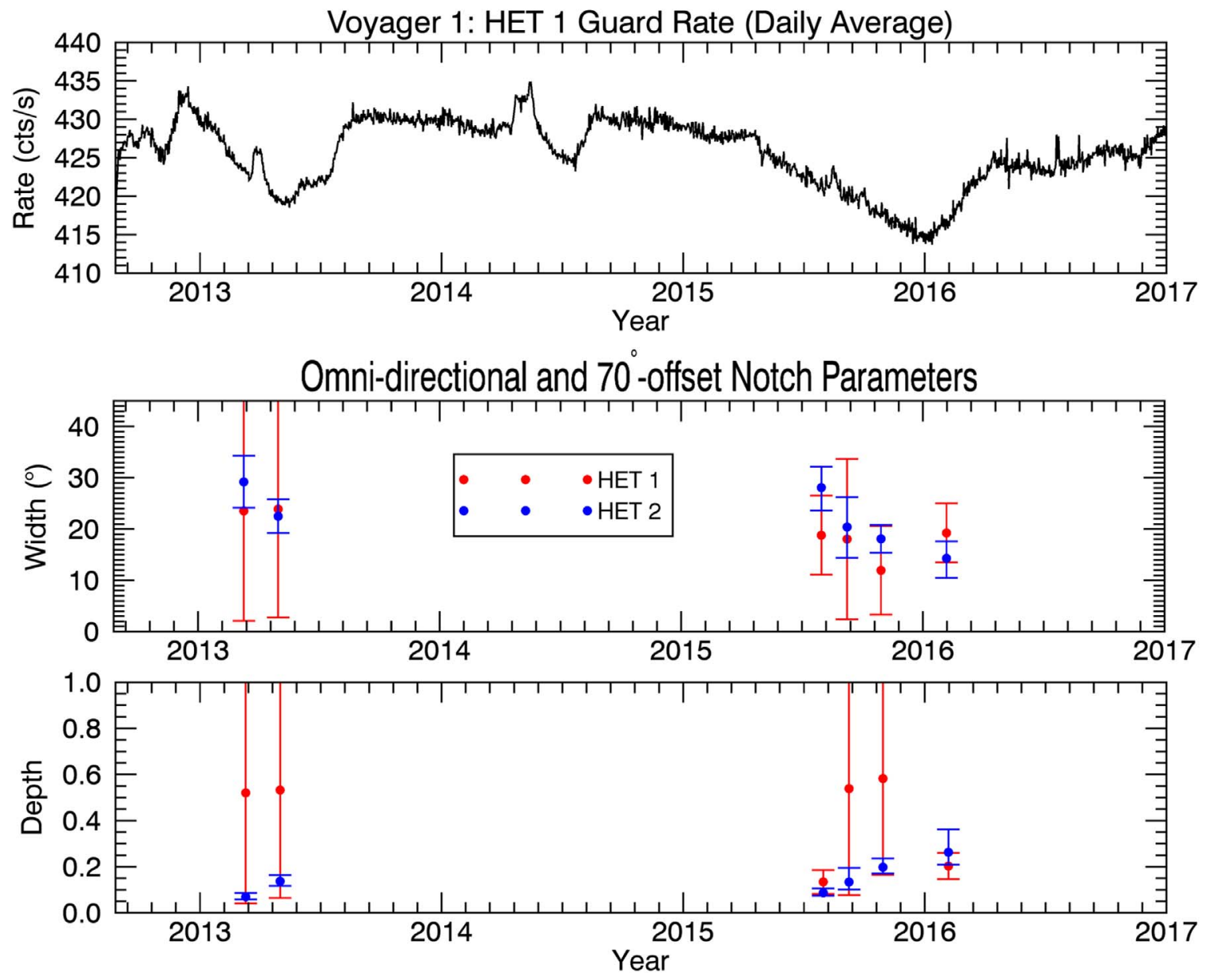

Figure 8. Widths and depths predicted from the intersection of omnidirectional and 70 ${ }^{\circ}$-offset simulations for HET 1 (red) and HET 2 (blue) incorporating the alternative fields listed in Table 6 of Appendix B.

we note that preserving the observed notch areas $\left(\delta_{\text {omni }}\right.$ and $\delta_{70^{\circ}}$ ) causes width and depth to vary inversely proportionally to each another. In other words, the wider the notch, the shallower the depth.

In Figure 9 we compare HET 1's roll maneuver notch parameters to the results from HET 2's $70^{\circ}$-offset and omnidirectional response function curves. The independently acquired results from these two approaches show agreement, and HET 1 and 2's widths and depths are consistent with one another favoring a broad, shallow notch that is, on average, $22^{\circ}$ wide and $15 \%$ deep.

\section{Discussion}

\subsection{Anisotropy Formation via Magnetic Trapping}

To understand the underlying physics of the pitch angle anisotropy, we must consider both its spatial formation and temporal evolution. The cosmic rays that are considered throughout this work are primarily on the order of several hundred megaelectron volts (Cummings et al. 2016) and are observed in the LISM where their scattering mean free paths are very large (Ptuskin 2001). Additionally, magnetic fluctuations beyond the heliopause are very small (Burlaga et al. 2018). Therefore, it is reasonable to assume that particle energies remain constant as they follow slowly varying magnetic field lines. As such, we can describe the anisotropy's spatial formation through magnetic trapping, which arises from the conservation of the first adiabatic invariant:

$$
\frac{\sin ^{2} \alpha(x)}{|B|_{x}}=\frac{\sin ^{2} \alpha_{o}}{|B|_{o}}=\text { const. }
$$

for a distance $x$ along the field line and values $\alpha_{o}$ and $|B|_{o}$ at the point of observation. As particles with pitch angles $\alpha(x)$ encounter stronger fields, their pitch angles increase until they reach a mirror point $\left(x_{m}\right)$-where $\alpha_{m}=90^{\circ}$ and $|B|_{m}=\frac{|B|_{o}}{\sin ^{2} \alpha_{o}}$-after which the parallel component of the Lorentz force causes particles to reverse direction and move back toward regions of lower $|B|$. Therefore, if a weak magnetic field $\left(|B|_{w}\right)$ is bounded by a strong field in either direction along the field line $\left(|B|_{s}\right.$-although the two directions could differ in strength), the largest pitch angles to arrive in the weaker field region $\left(\alpha_{w}\right)$ will be determined by:

$$
\alpha_{w}=\sin ^{-1}\left( \pm \sqrt{\frac{|B|_{w}}{|B|_{s}}}\right)
$$

for particle velocities parallel $(+)$ and antiparallel $(-)$ to the field. Hence, $\alpha_{w}<90^{\circ}$ because $\sqrt{\frac{|B|_{w}}{|B|_{s}}}<1$, resulting in a pitch angle gap in the weak field region near $90^{\circ}$ with a total width of:

$$
w=2\left(90^{\circ}-\alpha_{w}\right) .
$$



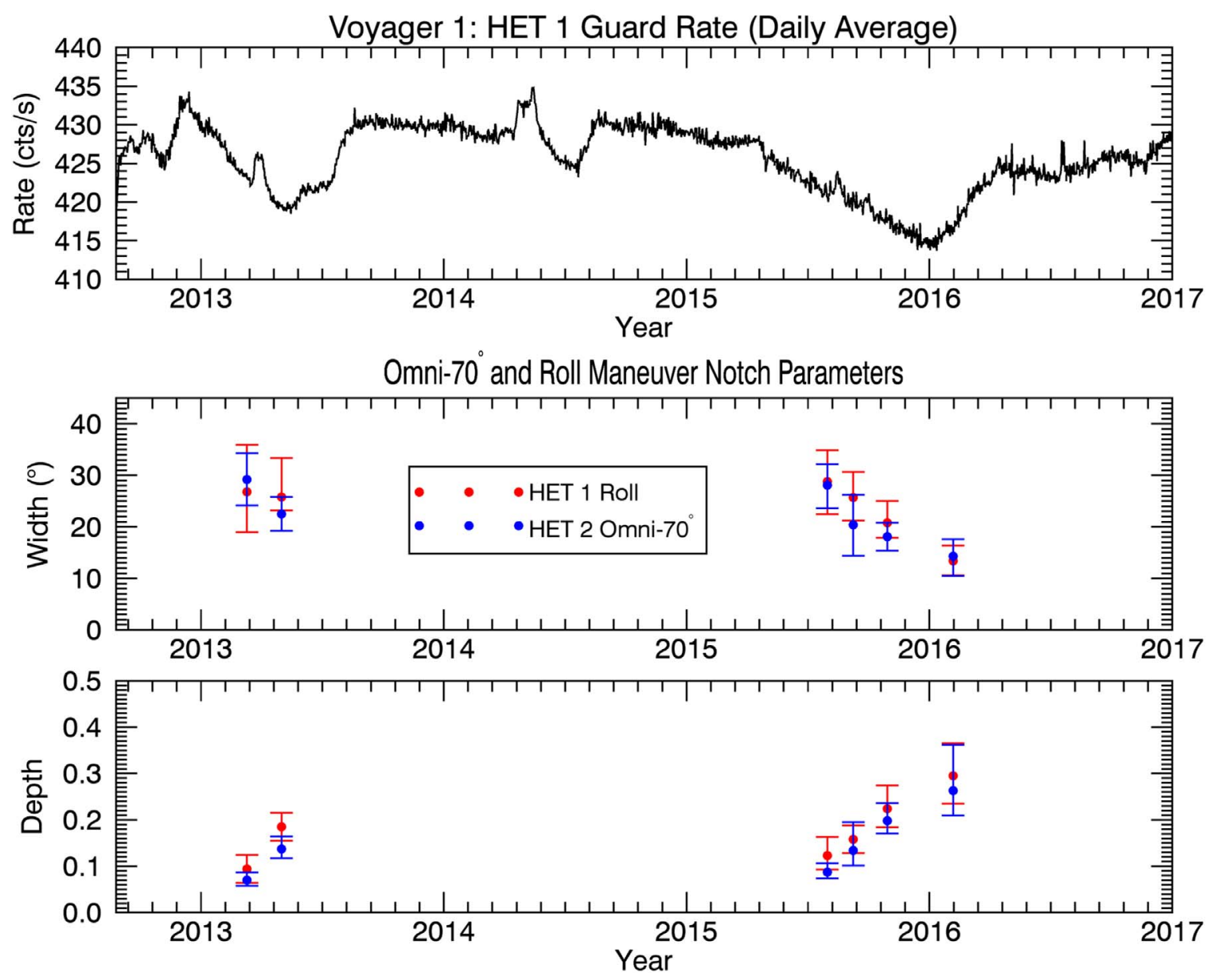

Figure 9. Comparison of HET 1 roll maneuver widths and depths (red) to independently acquired HET 2 omni- $70^{\circ}$ results (blue).

Because trapping occurs only within a region bounded by stronger fields, what, then produces this requisite field geometry?

\subsection{Trapping Mechanisms}

Figure 10 illustrates three possible trapping scenarios. The boundaries of a quasi-perpendicular shock could, in theory, supply the necessary geometry for trapping (Figure 10(a)). According to Kóta \& Jokipii (2017), particles trapped downstream (in the shocked plasma) undergo cooling as they interact with adiabatically expanding magnetic fields (see Figure 1 of Kóta \& Jokipii 2017). Those with pitch angles nearest to $90^{\circ}$ stay trapped for the longest amount of time, experience the greatest energy loss, and therefore contribute the most to reductions in GCR flux (see Figures 3 and 5 of Kóta \& Jokipii 2017).

Many aspects of this model are compelling. For example, Kóta \& Jokipii (2017) predict decreasing trends in the GCR flux for a $\mu=0.00$ to 0.25 pitch angle segment and not in other segments $(\mu>0.25)$ (see their Figures 3 and 5). This translates to a maximum notch width of $w=2 \times\left(90^{\circ}-\cos ^{-1}(0.25)\right)=29^{\circ}$. Our analysis agrees. Model \#2 prescribed a $\sim 29^{\circ}$-wide notch for 2013-67 (Table 10 in Appendix B); this is the interval nearest to the shock that Kóta and Jokipii used to inform their model, which was observed by Voyager on 2012-335 (see Burlaga \& Ness 2016).

The global structure of the heliopause likely also influences the trapping geometry, as shown in Figures 10(b) and (c). We argue here that the draping and compression of the interstellar magnetic field around the heliopause, as demonstrated by $I B E X$, naturally creates a permanently compressed field region that could also serve as a mirror point for the trapped particles. The steady-state field compression likely occurs where the field is most tangent to the heliopause: at the $I B E X$ ribbon. Indeed, according to the model of Zirnstein et al. (2016), the field near the heliopause is strongest in the direction of the ribbon and is $20 \%$ stronger (E. Zirnstein 2019 , private communication) than the $0.48 \mathrm{nT}$ average field seen by Voyager (Burlaga \& Ness 2016).

As Figure 10(b) illustrates, the situation described by Kóta \& Jokipii (2017) and the existence of the naturally steady-state compression are not necessarily exclusive, depending on the strong field boundary conditions. For example, if $|B|_{\mathrm{s}}(\mathrm{HP})<|B|_{\mathrm{s}}(1)$ and $|B|_{s}(2)$, particles will remain trapped completely within the disturbance (as in Figure 10(a)). Alternately, if $|B|_{\mathrm{s}}(1)<|B|_{\mathrm{s}}(\mathrm{HP})$, the trapping region could extend toward the heliopause compression region. We also note that the boundary conditions could change over time; even if the trapped particles are initially contained within the disturbance, they could eventually mirror at $|B|_{\mathrm{s}}(\mathrm{HP})$ as the transient weakens over time or distance.

Figure 10(c) shows another possible scenario where Voyager remains in the unshocked plasma without locally encountering the disturbance itself. If $|B|_{\mathrm{s}}(1)<|B|_{\mathrm{s}}(2)$, Voyager could still observe the cooled particles as they escape from the disturbance. Alternatively, anisotropies could also form if the locally trapped particles (e.g., those near $90^{\circ}$-pitch angle) are affected by some (perhaps unrelated) microscopic or 


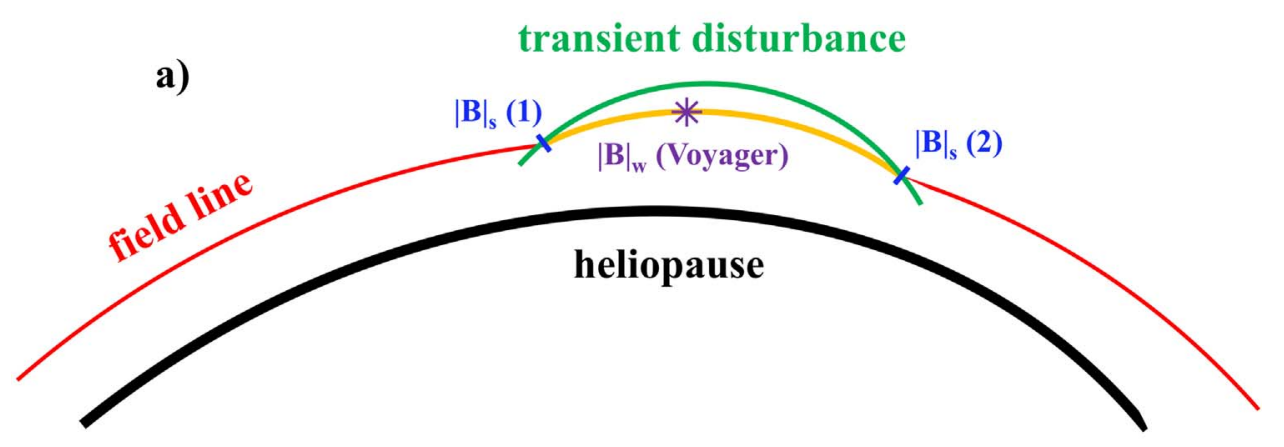

transient disturbance
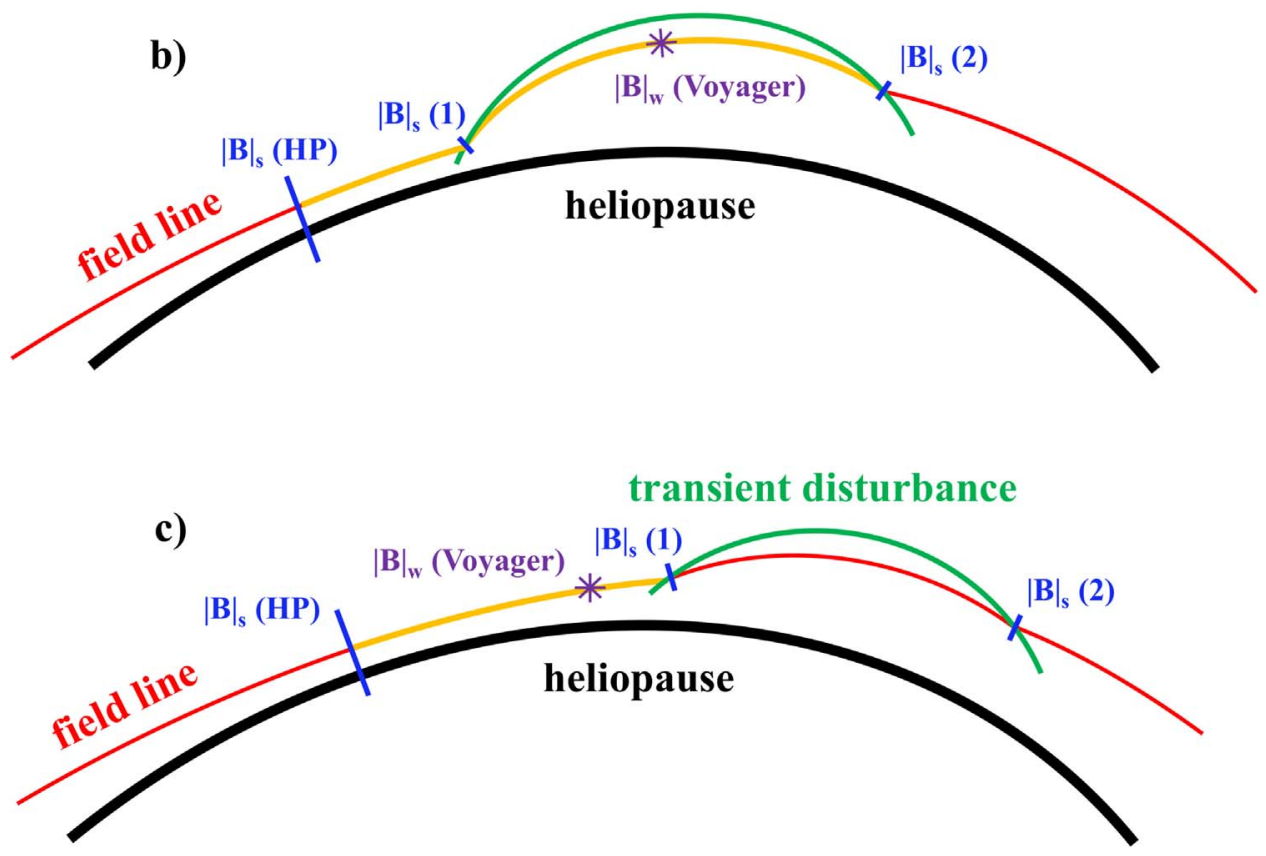

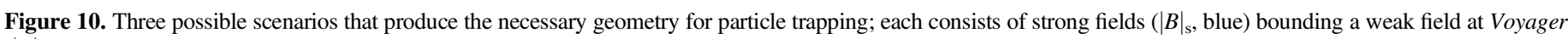

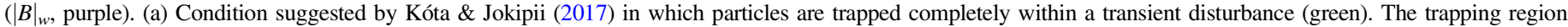

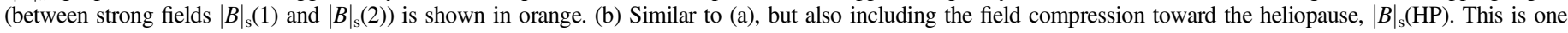

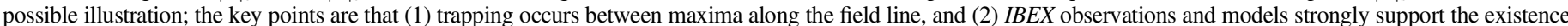

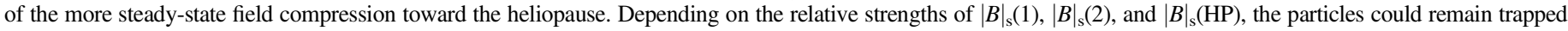

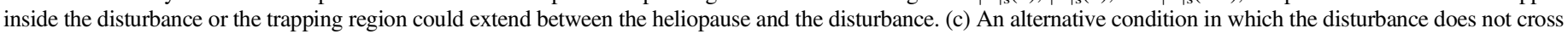
Voyager, but particles are trapped between the heliopause compression, $|B|_{\mathrm{s}}(\mathrm{HP})$, and the disturbance (the stronger of $|B|_{\mathrm{s}}(1)$ or $\left.|B|_{\mathrm{s}}(2)\right)$.

macroscopic energy loss mechanisms, such as expanding fields produced by an inward motion of the heliopause (Washimi et al. 2011, 2017).

\subsection{Physical Interpretation of Pitch Angle Notches}

We now consider how particle trapping relates to the notch's width and depth. Suppose Voyager is on a field line that is filled with an isotropic distribution of particles and has an enhanced strength near the heliopause compression but no other local maxima. So long as these initial conditions are satisfied, Voyager will continue to observe particles at all pitch angles with constant intensity. When a compression passes by, it generates additional local maxima. This change of boundary conditions isolates part of the pitch angle distribution (those near $90^{\circ}$ ) so that the affected particles are no longer replenished by the surrounding vast cosmic ray reservoir. The notch's width is a measure of this isolation. However, trapping alone is not sufficient to produce a notch; either particles must leak out of the trap more quickly than it is refilled or they must experience some sort of energy loss that translates to a reduction in intensity. In other words, the notch represents a combination of both width and depth, trapping, and intensity change.

The notch's width reflects the extent of the affected particle pitch angle distribution and is a measure of the ratio of the strong and weak fields. Combining Equations (2) and (3) leads to:

$$
\begin{aligned}
\Delta|B|_{n} & =\left(|B|_{s}-|B|_{w}\right) /|B|_{s}=\cos ^{2}(\alpha) \\
& =\cos ^{2}\left(90^{\circ}-w / 2\right)
\end{aligned}
$$

where $\Delta|B|_{n}$ is the change in the ambient field that is required to produce a notch of width $w$. A notch that is too narrow could potentially be erased by turbulent fluctuations in the steady-state magnetic field. In the LISM, Voyager observes fluctuations of $\sim 2 \%$ over several-week timescales (Burlaga et al. 2015, 2018); given that the GCR anisotropic decreases endure for many months at a time, this serves as a lower limit to $|B|_{n}$. Additionally, a notch 


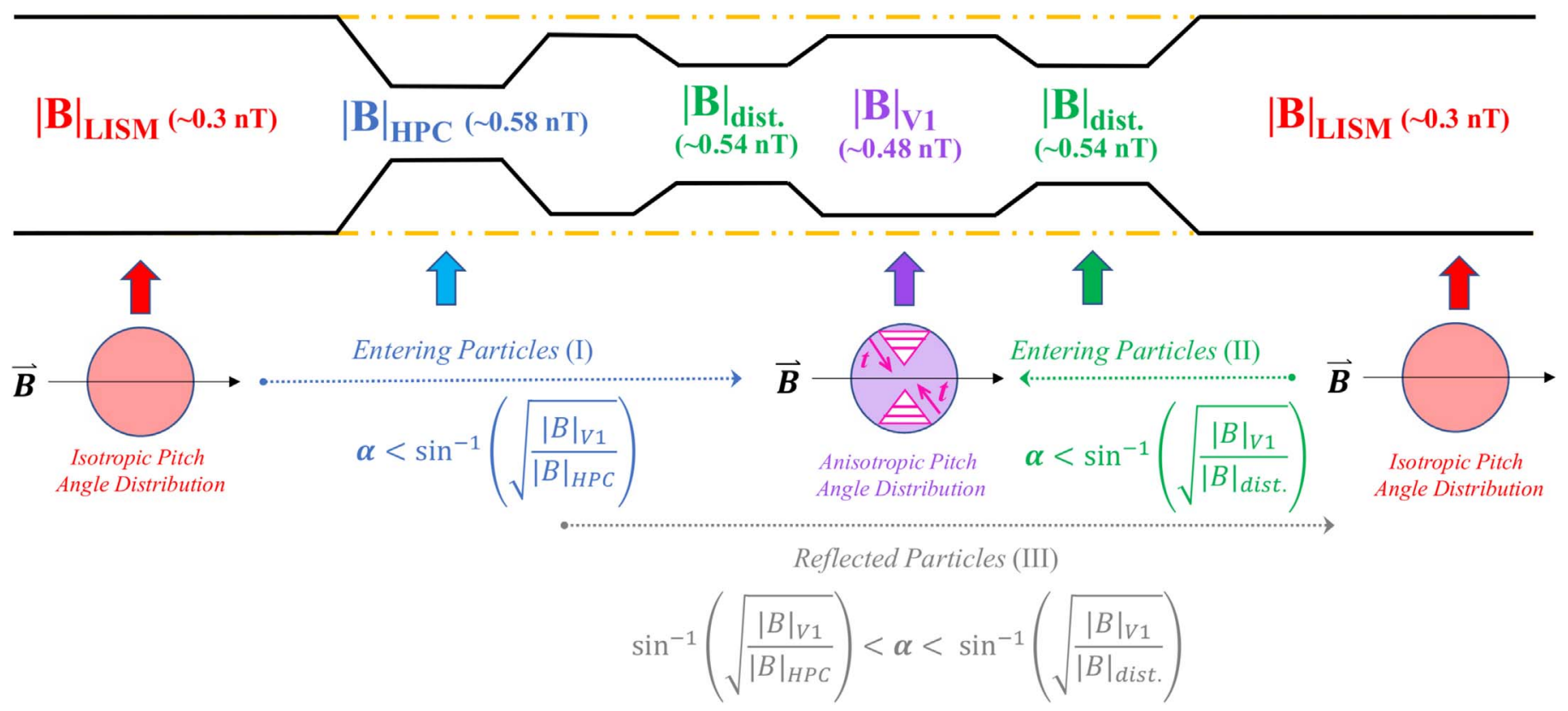

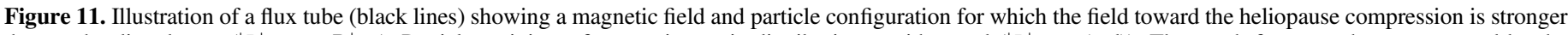

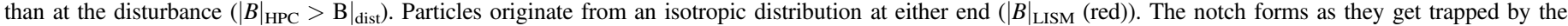

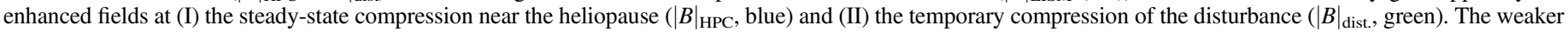

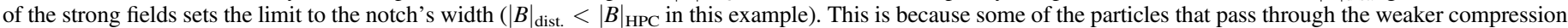

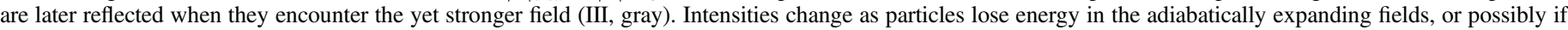

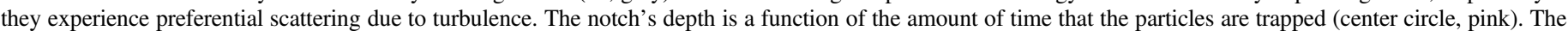

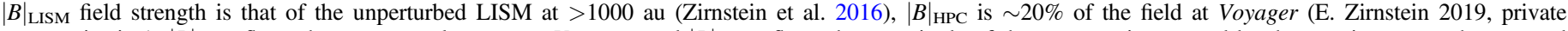

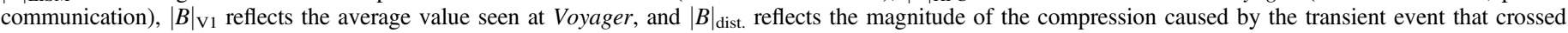
Voyager on 2014-237 (Burlaga \& Ness 2016).

should not require changes in the field that are larger than we observe. For example, in 2014, Voyager encountered a traveling shock that produced a $\sim 12 \%$ enhancement of the field (Burlaga \& Ness 2016); this provides an upper limit to $\Delta|B|_{n}$. Thus, we expect the notch widths from our simulations to reflect field changes of $\sim 2 \% \lesssim \Delta|B|_{n} \lesssim \sim 12 \%$. Averaging Model \#2's six intervals yields a notch that is $22^{\circ}$ wide and $15 \%$ deep (Section 4.2), so $\Delta|B|_{n}=4 \%$. Because $2 \%<4 \%<12 \%$, our average broad, shallow notch implies changes in $|B|$ that are reasonably consistent with the observations.

The notch's depth reveals how magnetic trapping leads to changes in the GCR intensity. Two likely contributors include adiabatic cooling and scattering. When Voyager is downstream of the shocked plasma, adiabatic cooling is likely the dominant energy loss mechanism (Figures 10(a) and (b)) as suggested by Kóta \& Jokipii (2017). Because of the negative spectral index of the relevant-energy GCRs in the LISM (Cummings et al. 2016), loss in particle energy translates to reduced intensity, per Liouville's theorem (see for example, Kóta \& Jokipii 2017). A partially filled notch could also be indicative of some nonadiabatic process. For example, the local turbulence-especially if it differs from its surroundings - might affect the rate at which particles escape (or enter) the trapping region. The interplay between turbulence and cooling might also provide clues as to why these notches are long-lasting, yet "mostly filled." The relative amount that adiabatic cooling or scattering processes may contribute to (or hinder) the notch's formation merits further study. The depth likely grows as a function of the time that the particles spend in the trap. Again, we emphasize that our best-fit notches are mostly filled — on average only $15 \%$ deep — and the changes we measure in the omnidirectional intensity distribution are small-less than $4 \%$ - so only small amounts of cooling or scattering are necessary to produce the effects that we observe.

Figure 11 shows a schematic $B$-field diagram of a trapping scenario where the fields of the disturbance are weaker than the compression toward the heliopause. Again, Voyager must be in the weak field region in order to observe the anisotropy, and the region must be bounded by stronger fields along those field lines. The compressed field at the heliopause likely differs in magnitude from the enhanced field at the disturbance, so if both of these contribute to the trapping, it is the weaker of these strong fields that ultimately determines the notch's width.

Figure 12 shows the sequence of LISM shock transient events observed by Voyager on multiple instruments. GCRs periodically undergo roughly month-long intensity enhancements (e.g., Figure 12(a), near $\sim 2013.2$ and $\sim 2014.3$ ) that are reminiscent of the shorter-lived particle spikes produced at the foreshock of interplanetary shocks, as modeled by Jokipii \& Kóta (2014) and noted by Gurnett et al. (2015). Because of the particles' high energies, these are the first indications of the transients to arrive at Voyager. Next, Voyager 1's PWS observes emissions from electron plasma-beam instabilities, which also occur upstream of the disturbances (Gurnett et al. 2015; horizontal bars in Figure 12(b)). Finally, several weak, smooth, thick disturbances cross Voyager and are measured by the magnetometer (Burlaga \& Ness 2016; vertical lines in Figures 12(a) and (b)).

We compare the trends of our model to the observations (Figure 12) to evaluate our physical interpretation of the pitch angle notches. Table 2 contains a listing of Model \#2's results, along with an estimate of the magnitude of the strong field $\left(\left.B\right|_{s}\right)$ that is involved in producing the various notches. These estimates 

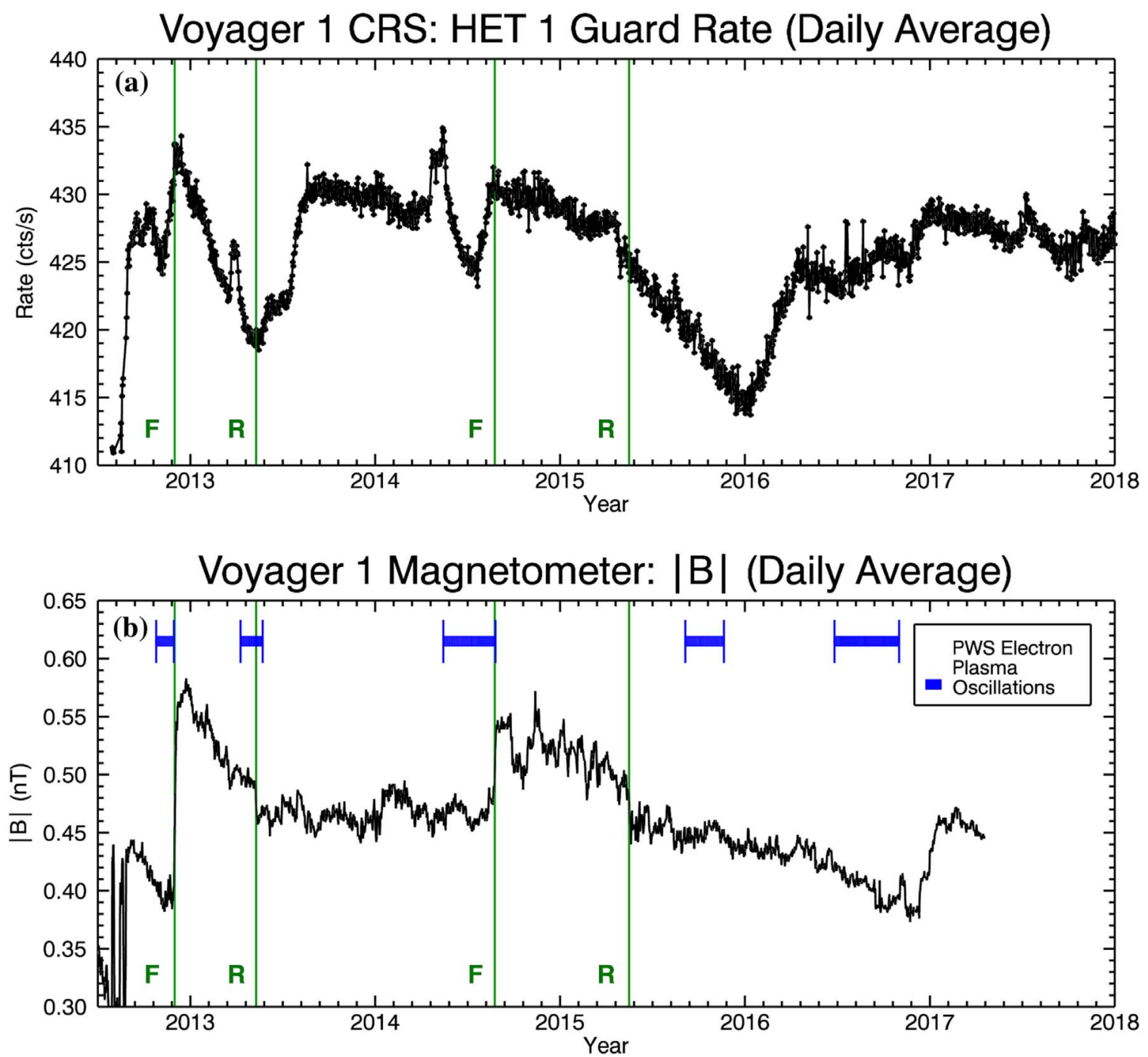

Figure 12. Comparison of shock transient and GCR anisotropy events observed by Voyager 1 in the LISM. (a) HET 1's omnidirectional guard rate ( $\gtrsim 20$ MeV; proton-dominated) with vertical lines indicating forward and reverse disturbances observed by Voyager 1's magnetometer (see Burlaga \& Ness 2016). (b) Magnetometer $B$-field strength vs. time with vertical lines indicating the disturbances as in panel (a) (from the magnetometer's publicly available data: https:// omniweb.gsfc.nasa.gov/coho/form/voyager1.html). The horizontal blue bars indicate the timing of emissions from electron plasma oscillations recorded by PWS (see Gurnett et al. 2013, 2015 for further details). The time periods shown reflect those of the $3.11 \mathrm{kHz}$ channel electric field measurements (from publicly available PWS data: http://www-pw.physics.uiowa.edu/voyager/data/).

are derived from Equation (4) by substituting Voyager's local magnetic field measurements for the weak field $\left(|B|_{w}=|B|_{\text {obs }}\right)$.

The largest predicted $|B|_{s}$ in Table 2 occurs during the 201367 epoch, and its $\sim 0.55 \mathrm{nT}$ value is very close to the reported maximum field strength $(\sim 0.56 \mathrm{nT})$ of the magnetometer's 2012-335 shock transient event ( 2012.9 in Figure 12(b); see also Figure 3 in Burlaga \& Ness 2016). During the 2015-208 epoch, $|B|_{s} \sim 0.49 \mathrm{nT}$, which agrees with the $0.494 \mathrm{nT}$ field observed at the time of the preceding 2015-137 reverse shock ( 2015.4 in Figure 12(b); see also Figure 5 in Burlaga \& Ness 2016). Because our calculated strong field seems consistent with the strength of passing disturbances, the steady-state field toward the ribbon is likely stronger than the temporary fields for these periods.

Table 2's trends in widths and depths (per episode) make sense in relation to the disturbances' temporal behavior. We expect the magnetic field (hence the widths) to weaken toward the anisotropy minima (intervals 2013-120 and 2016-21 in Table 2; 2013.35 and 2016.0 in Figure 12(a)) because the field at the transients likely weakens as the shock moves further out. Additionally, we expect the depth - and therefore the magnitude of the anisotropy - to grow as a function of longer cooling and scattering times, so long as the particles remain trapped.

There are local indications that adiabatic cooling plays an important role in the anisotropy's growth. For example, in 2013, the field weakens on the same timescales that the anisotropy develops (see $\sim 2012.9$ to $\sim 2013.35$ in Figure 12). The 2015 episode shows similar, although not identical, behavior because its development occurs in a two-step process (from $\sim 2014.65$ to $\sim 2015.35$ and $\sim 2015.35$ to 2016.0 ). The 2014 episode, however, is an exception; it occurs during a time when the local fields appear neither expanded nor compressed, and it is not preceded by an obvious disturbance. Perhaps the field geometry is similar to Figure 10(c) and Voyager senses cooled particles escaping from the shock or some different energy-loss mechanism is affecting the locally trapped particles. 
Table 2

$B$-field Variations $\left(\Delta|B|_{n}\right)$ Required to Produce a Notch of a Given Geometry, Informed by Model \#2's Parameters

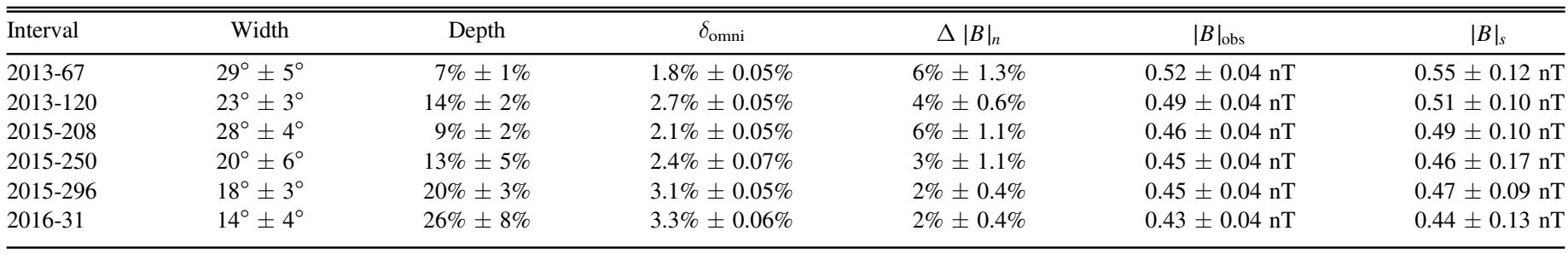

Note. $|B|_{\text {obs }}$ is the strength of the local field measured by Voyager. $|B|_{s}$ represents the magnitude of the strongest field in the trapping configuration. The latter is determined by substituting the observed field $\left(|B|_{\mathrm{obs}}=|B|_{w}\right)$ into Equation (4) (see also Appendix B, Tables 5, 6 and 10).

The onset of each recovery seems to occur when either the trap has dissipated or Voyager has moved beyond the trapping region. For example, the 2013 episode recovers while the local field no longer weakens but appears to fluctuate about $\sim 0.46 \mathrm{nT}$ ( 2013.35 to $\sim 2013.6$ in Figure 12). For the 2015 episode, Table 2 shows that the strong field weakens to roughly the observed value by 2016-31 $\left(\left.B\right|_{\text {obs }} \sim|B|_{s}\right)$, near the GCR intensity minimum ( 2016.0 in Figure 12(a)). However, beyond the minimum (Figure 12), the local magnetic field continued to decrease below average through the end of 2016. Perhaps during this recovery, Voyager's field lines were still connected to the shock, but no longer connected to the strong field toward the ribbon, or maybe some sort of change occurred near the heliopause.

\subsection{Summary and Conclusion}

We have provided evidence that the GCR pitch angle anisotropy observed by Voyager 1 in the LISM is characterized by a broad, shallow, mostly filled notch caused by particles that are missing near $90^{\circ}$ in an otherwise uniform pitch angle distribution. We suggest that the notch forms in a trapping region that, in addition to being affected by temporarily compressed fields from traveling disturbances, could also be affected by the presence of a steady-state enhanced magnetic field near the heliopause. IBEX observations support the existence of the former, at the heliopause near the ribbon. Regarding the latter, all of Voyager's working instruments have detected signatures of several weak, solar-induced LISM transients. The notch's width correlates with the ratio of the local field at Voyager and the remote field of the compressions. The notch's depth relates to the anisotropy's growth as a function of time and is at least partially due to adiabatic cooling. Topics that merit further investigation include the following: (1) investigating the role that turbulence might also play in the anisotropy's development, (2) examining the factors that contribute the anisotropy's magnitude and recovery, (3) understanding how the anisotropy behaves as a function of particle species and energy, and (4) exploring how the steady-state compression toward the ribbon might additionally affect the trapped particle population. We look forward to continuing our evaluations of Voyager's in situ observations together with IBEX's global measurements, as this will potentially lead to greater insight about the heliosphere and its interaction with the interstellar medium.

We thank E. Roelof for first suggesting particle trapping as a means for generating the anisotropy, R. Decker and the LECP team for providing background-corrected data, and E. Zirnstein for providing model-based information about the field enhancement toward the heliopause. This work was supported

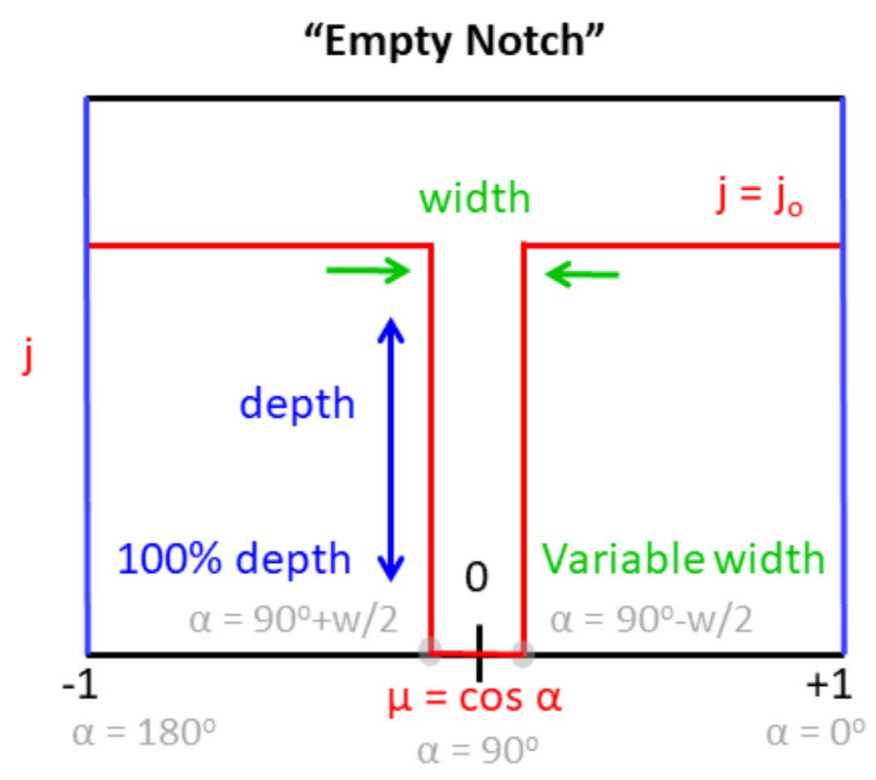

Figure 13. Diagram of notch Model \#1.

by NASA grant No. NNN12AA01C. J.S.R. and D.M. were also supported by the Interstellar Boundary Explorer (IBEX) mission, which is part of NASA's Explorer Program.

\section{Appendix A Model \#1: Empty Notch}

Model \#1 assumes negligible scattering and represents the notch as a complete dropout of particles within a range of pitch angles characterized by variable width and $100 \%$ depth (see Figure 13). These assumptions enable us to efficiently fit the simulated directional response functions to data using a single "effective width" parameter.

\section{A.1. Omnidirectional Response Function}

The omnidirectional intensity $(J)$ is represented by the general expression:

$$
J=2 \pi \int_{-1}^{1} j(\mu) d \mu
$$

where $j$ denotes directional particle intensity and $\mu$ is related to pitch angle, $\alpha$, by $\mu=\cos \alpha$. For an isotropic distribution, $j=j_{0}$ and is constant, so $J=4 \pi j_{0}$. For a distribution with a notch, the missing particle intensity $\left(J_{n}\right)$ is given by the integral 


\section{Simulated Particles Entering HET 1 A-end \\ Pitch Angle vs Telescope Clock Angle: 2015-310 Roll Epoch}

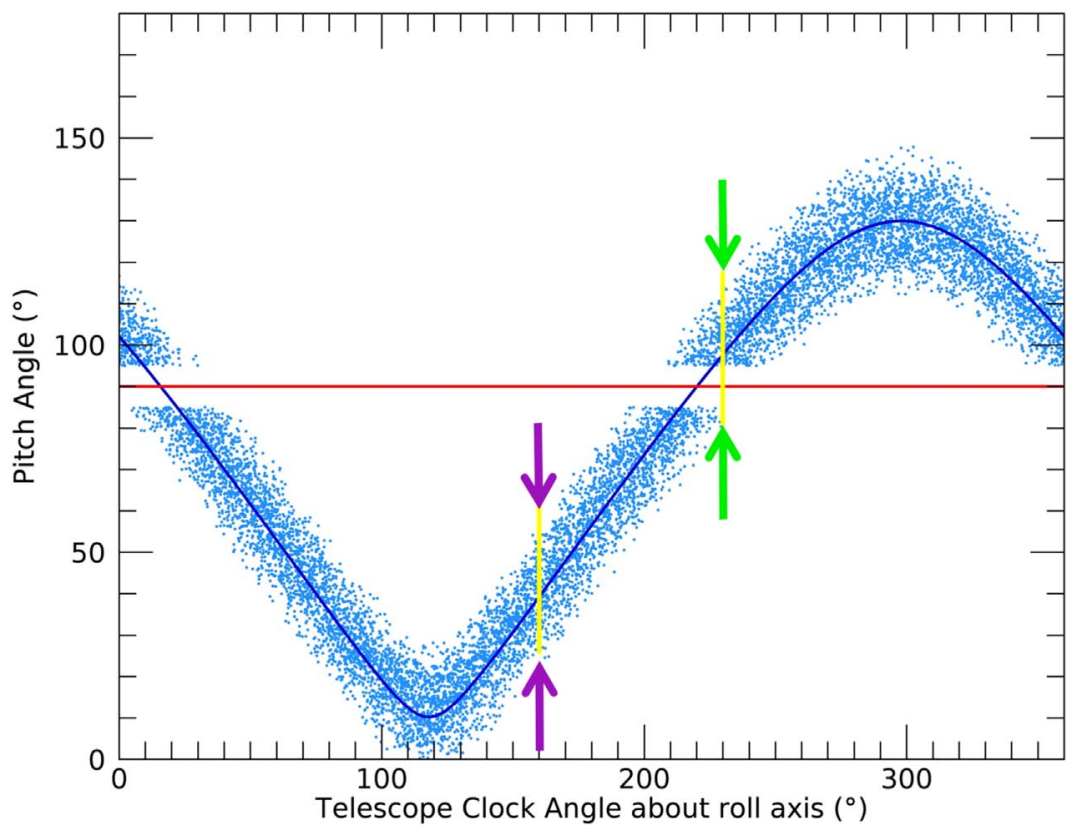

Figure 14. Pitch angle vs. telescope clock angle (measured from $\hat{N}$ toward $\hat{T}$ ) view of the 2015-310 roll maneuver Monte Carlo simulation for particles entering HET 1 's A-end, shown with a $10^{\circ}$-wide notch. The magnetic field direction during this time was $(0.156,-0.381,0.202) \mathrm{nT}$ in $R, T, N$ (from the magnetometer's publicly available data: https://omniweb.gsfc.nasa.gov/coho/form/voyager1.html). Simulated particles (blue dots) fall within a $\sim 40^{\circ}$-wide band, as defined by the telescope's opening angle. HET 1's nominal boresight is centered at $160^{\circ} 9$ clock angle and 40.5 pitch angle; its normal field of view (indicated by the purple arrows) does not overlap with $90^{\circ}$ pitch angle (red horizontal line). However, when the HET 1 boresight passes through $\sim 17^{\circ}$ and $\sim 219^{\circ}$ clock angle during the $2015-310$ roll maneuver, the notch is directly centered in its field of view; therefore, a measurable count rate reduction is observed (see Figure 16(a)). We note that the clock angle difference between the two pitch angle minima is close to, but not at, $180^{\circ}$. This is because the $B$-field is mostly in the $\hat{N}-\hat{T}$ plane, but not quite perpendicular to the spacecraft's rotation axis $(\hat{R})$. HET 1 's $70^{\circ}$-offset boresight is at $230^{\circ} 9$ clock angle and 98.5 pitch angle, so its field of view also overlaps with the anisotropy during the offsets (green arrows).

\section{Pitch Angle vs Telescope Clock Angle: 2015-310 Roll Epoch}

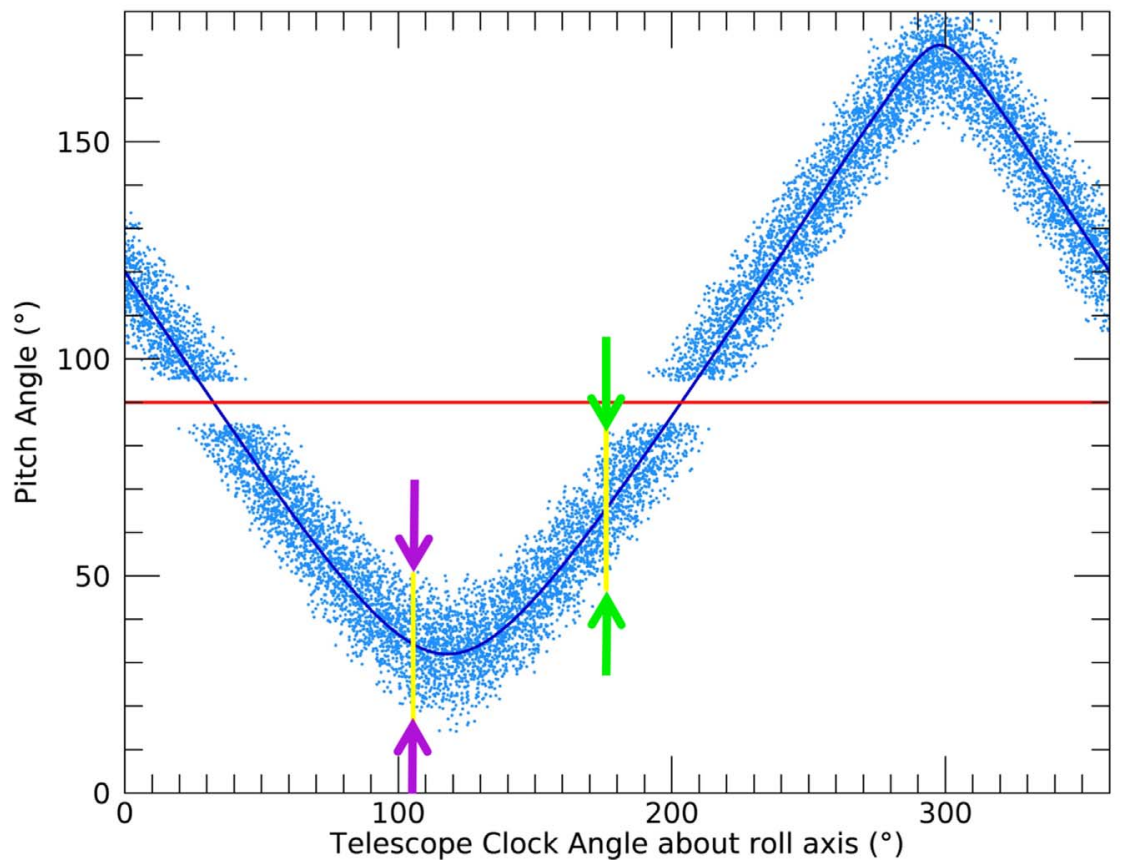

Figure 15. Similar to Figure 14, but for particles entering HET 2's B-end. HET 2's normal field of view (purple arrows) does not overlap with $90^{\circ}$ pitch angle (red horizontal line) because its normal boresight is centered at $107^{\circ} .1$ clock angle and $33^{\circ} .7$ pitch angle. HET 2 's $70^{\circ}$-offset boresight for 2015-310 is at $177^{\circ} .1$ clock angle and $66^{\circ} .4$ pitch angle, placing HET 2's field of view (green arrows) at the edge of the anisotropy; it may see an intensity decrease if the notch is wide enough. 

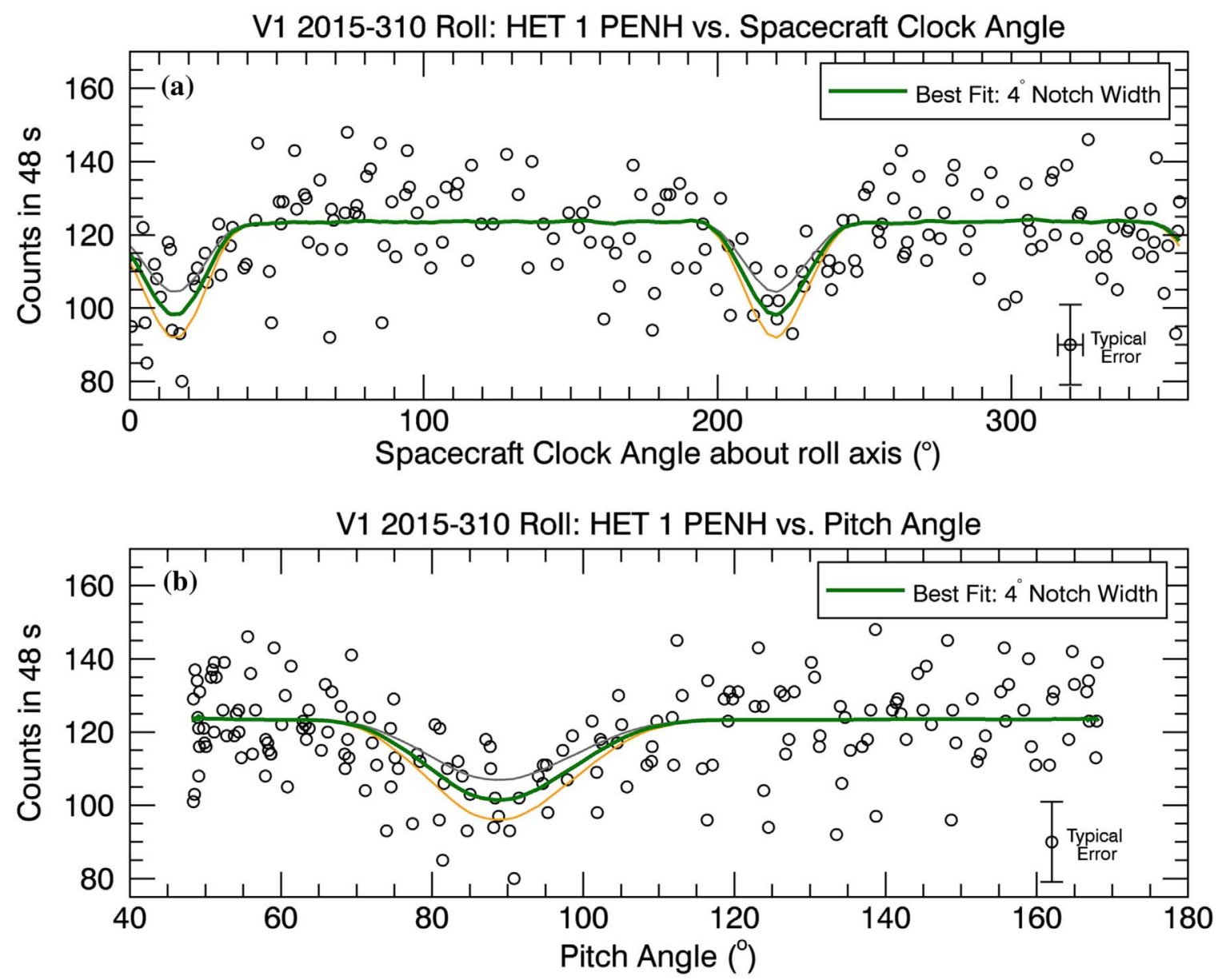

Figure 16. HET 1's bidirectional PENH rate ( $770 \mathrm{MeV}$; proton-dominated) vs. clock angle (a) and pitch angle (b) during the 2015-310 roll maneuver. The magnetic field direction during this time was $(0.156,-0.381,0.202) \mathrm{nT}$ in $R, T, N$ (from the magnetometer's publicly available data: https://omniweb.gsfc.nasa.gov/coho/ form/voyager1.html). The thick green solid line superimposed over the data represents the best-fitting notch roll response function produced by a Monte Carlo simulation with a width of $4.0 \pm 0.4$. Independent fits applied in clock angle space and pitch angle space yielded the same best-fit geometry. The thinner top (gray) and bottom (gold) lines represent $3^{\circ}$ and $5^{\circ}$-wide notches, respectively, plotted for visual reference. The horizontal line on the typical error reflects an 8.6 angular averaging within the $48 \mathrm{~s}$ data interval produced by the spacecraft as it rolls in clock space, while the vertical line reflects the statistical uncertainty in the number of counts. Count reductions appear broadened in both pitch angle and clock angle space, reflecting the $\sim 40^{\circ}$ opening angle of the telescope.

over the notch's effective width $w$ centered at $\alpha=90^{\circ}$ :

$$
J_{n}=2 \pi \int_{\cos (90 \circ+w / 2)}^{\cos \left(90^{\circ}-w / 2\right)} j \mu d \mu=4 \pi j_{n} \cos \left(90^{\circ}-w / 2\right) .
$$

Assuming negligible scattering implies that $j_{n}=j_{0}$, leading to the normalized "omnidirectional notch response function":

$$
\delta_{\text {omni }}=\frac{J_{n}}{J}=\cos \left(90^{\circ}-w / 2\right)=\cos \alpha .
$$

\section{A.2. Directional Response Functions}

First, we simulate a magnetometer roll maneuver by using a Monte Carlo simulation to calculate the pitch angle distribution of particles passing through HET 1 or HET 2 (detailed in the following section), accounting for each interval's observed magnetic field direction (e.g., Figures 14 and 15).

By excluding particles within an effective width centered about $90^{\circ}$ pitch angle and normalizing to observed rates, we produce a smooth width-dependent roll maneuver notch response function (e.g., Figure 16) and calculate its $\chi^{2}$ with respect to the $48 \mathrm{~s}$ data. After repeating the process for different widths, we minimize $\chi^{2}$ to acquire a best-fit notch for each roll interval.
Figure 16 shows simulated roll maneuver response function fits to observed bidirectional HET 1 PENH counts during the 2015-310 maneuver. The best fit was generated by a notch with an effective width of $4.0 \pm 0.4$. Fits in both clock angle (Figure 16(a)) and pitch angle (Figure 16(b)) space yield the same effective widths (to within \pm 0.05 or smaller) for all intervals. Because CRS telescopes view the anisotropy as a function of clock angle during the roll maneuvers, we report clock angle fits throughout this work.

Next, we calculate a $70^{\circ}$-offset rate reduction $\left(\delta_{70^{\circ}}\right)$ from the roll maneuver fit results by summing the counts in HET 1's simulated bidirectional response function (Figure 17(b)) with and without the notch cut. Figure 17(a) shows HET 1's observed count rate during $70^{\circ}$-offsets on days 2015-297 through 2015299 , a subset of the full sequence of offset maneuvers that took place nearest to the 2015-310 roll (see Figure 3). The observed $70^{\circ}$-offset reduction for this interval was $11.0 \% \pm 0.3 \%$ and the simulated value was $12.2 \% \pm 1.2 \%$. Figure 18 shows the same concept applied to HET 2.

Finally, we determine the omnidirectional intensity reduction $\left(\delta_{\text {omni }}\right)$ by applying the best-fit roll maneuver notch to Equation (7). For example, 2015-310's $4^{\circ} .0 \pm 0.4$ effective 

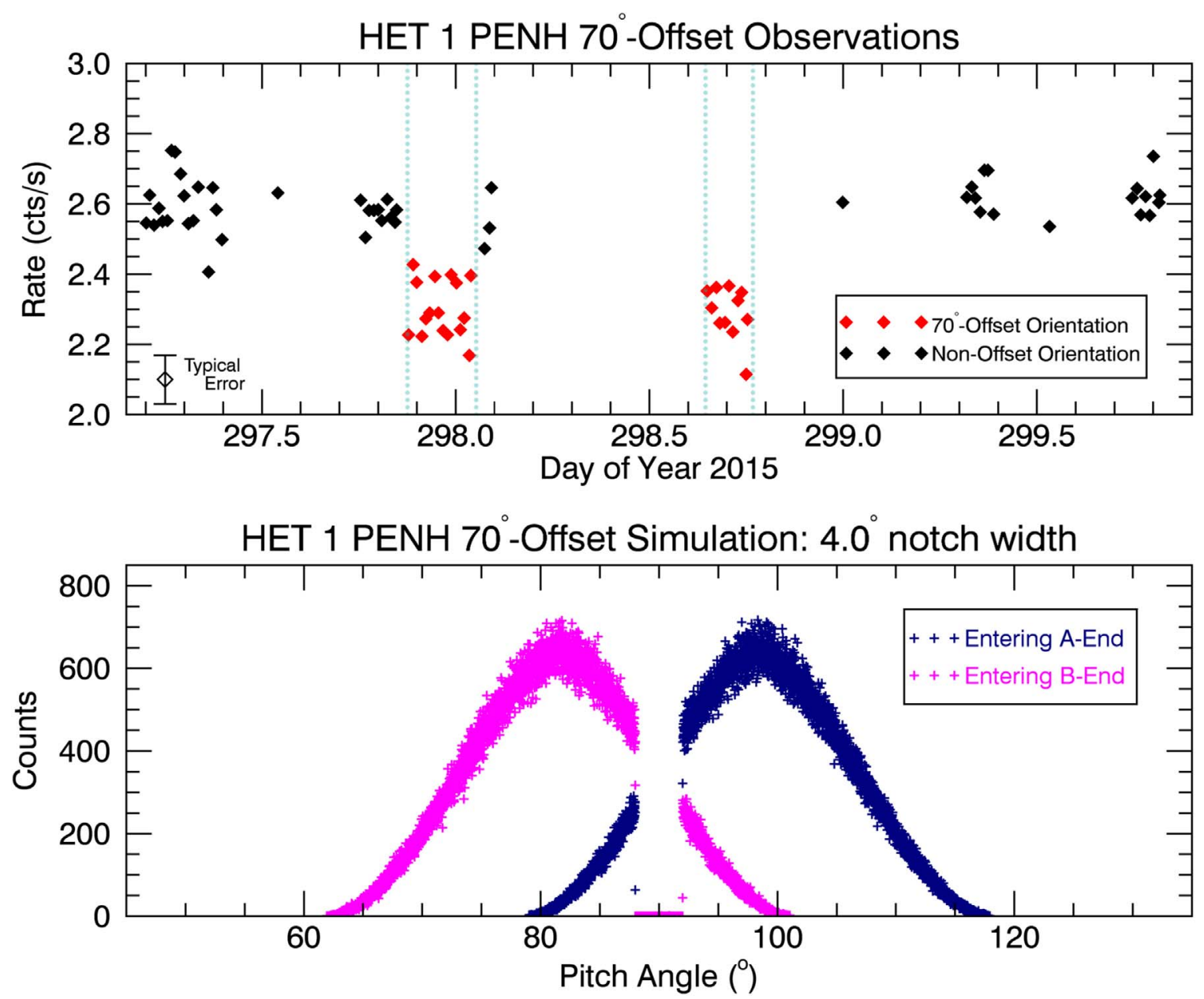

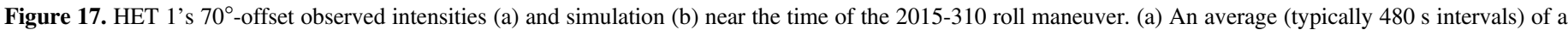

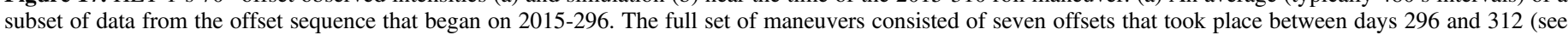

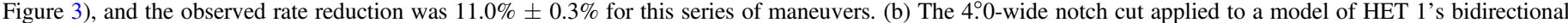
$70^{\circ}$-offset response function for particles entering the telescope's A-end (right; navy blue) and B-end (left; pink). The simulated reduction was $12.2 \% \pm 1.2 \%$.

notch width produces a $3.5 \% \pm 0.3 \%$ reduction, which is consistent with the observed value: $3.3 \% \pm 0.1 \%$ (comparable to the rates near $\sim 2015.8$ and $\sim 2013.85$ in Figure 1(b)).

\section{A.3. Monte Carlo Simulation Procedure}

We numerically simulate particles passing through HET 1 and HET 2 using the procedure outlined below. The directional observations throughout this work use each telescope's PENH rates, for which particles pass entirely through the detector stack. Because this coincidence mode reflects an integrated rate and does not discriminate among the various ions, we perform our particle selection in a manner that is independent of particle energy or species. To inform the telescope geometry, we use detectors $\mathrm{B}^{6}{ }^{6}$ and $\mathrm{C} 1$ (with radii of 1.596 and $1.739 \mathrm{~cm}$, respectively) spaced at a distance of $L=9.094 \mathrm{~cm}$ (measured from the top of one detector to the bottom of the other). See Stone et al. (1977) for more details about CRS telescopes.

\footnotetext{
6 The B detectors are curved, thin detectors. In the Monte Carlo simulation, B1 is modeled as flat and its spacing is defined relative to the bottom of its curvature. Although the curvature alters the path length of the particles and can affect the total energy loss, this has negligible effects on the integrated rates. Moreover, treating B1 as flat does not significantly alter a telescope's field of view.
}

1. Generate a particle on the first detector at a location uniformly randomly distributed in $x_{1}, y_{1}$.

2. Generate a random direction for the particle using a $\cos ^{2}(\theta)$ distribution.

3. Use these values to calculate the projected points in $x$ and y when a particle travels a distance $L$ in $\hat{z}$.

4. Keep only the projected points that pass through both detectors. Label these particle coordinates-defined with respect to the top detector-as $\left(p_{x}, p_{y}, p_{z}\right)$.

5. Convert particle coordinates to $R, T, N:\left(p_{x}, p_{y}, p_{z}\right) \rightarrow$ $\left(p_{r}, p_{t}, p_{n}\right)$.

6. Calculate the pitch angle by taking the dot product between the particle's coordinates and the observed $B$-field direction for a particular maneuver interval (in $R, T, N$ ).

7. For a given magnetic field direction and telescope viewing direction (different orientations for HET 1 and HET 2, for example), output information about the telescope orientation (clock angle, $\theta$ ) and particle pitch angles $(\alpha)$.

8. Simulate a roll maneuver by rotating the spacecraft about $\hat{R}$ in small clock angle increments over $360^{\circ}$ (in $R, T, N$ ) and repeat steps $1-7$ to accumulate the desired number of particles.

9. Simulate the $70^{\circ}$-offset data by fixing clock angle at $70^{\circ}$ —roughly a $70^{\circ}$-offset rotation about $\hat{R}$ (in $\left.R, T, N\right)$-and 


\section{HET 2 PENH $70^{\circ}$-Offset Observations}

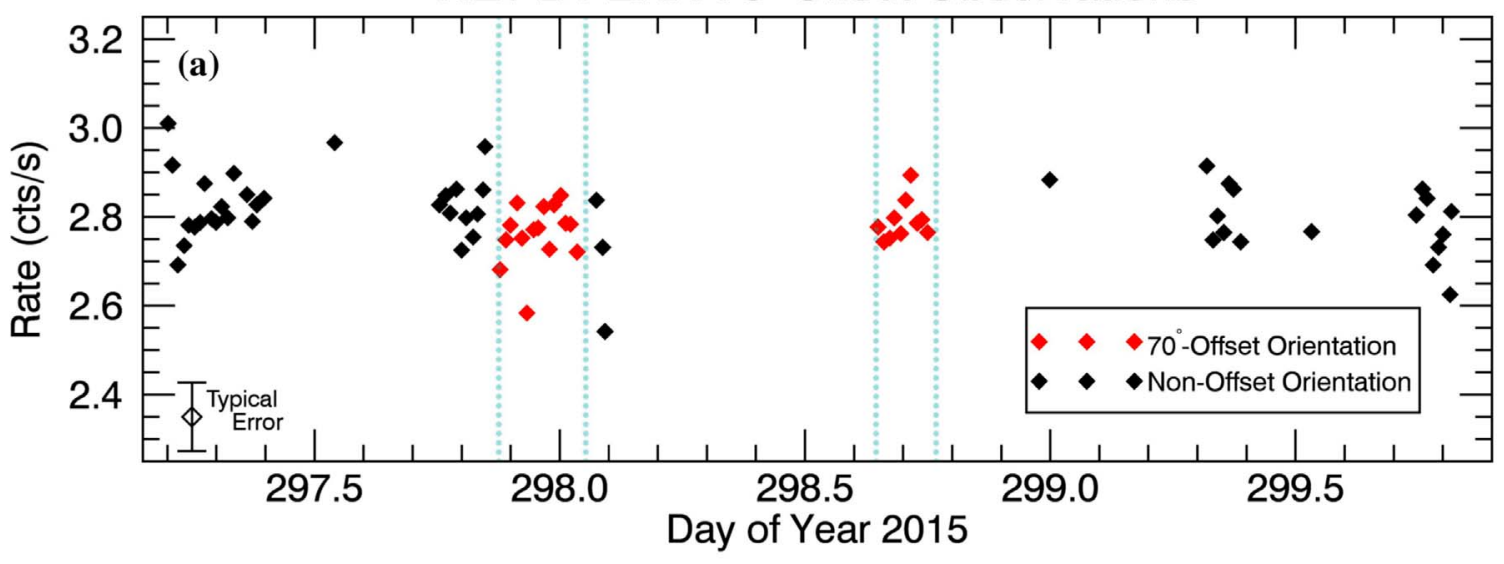

HET 2 PENH $70^{\circ}$-Offset Simulation: $4.0^{\circ}$ notch width

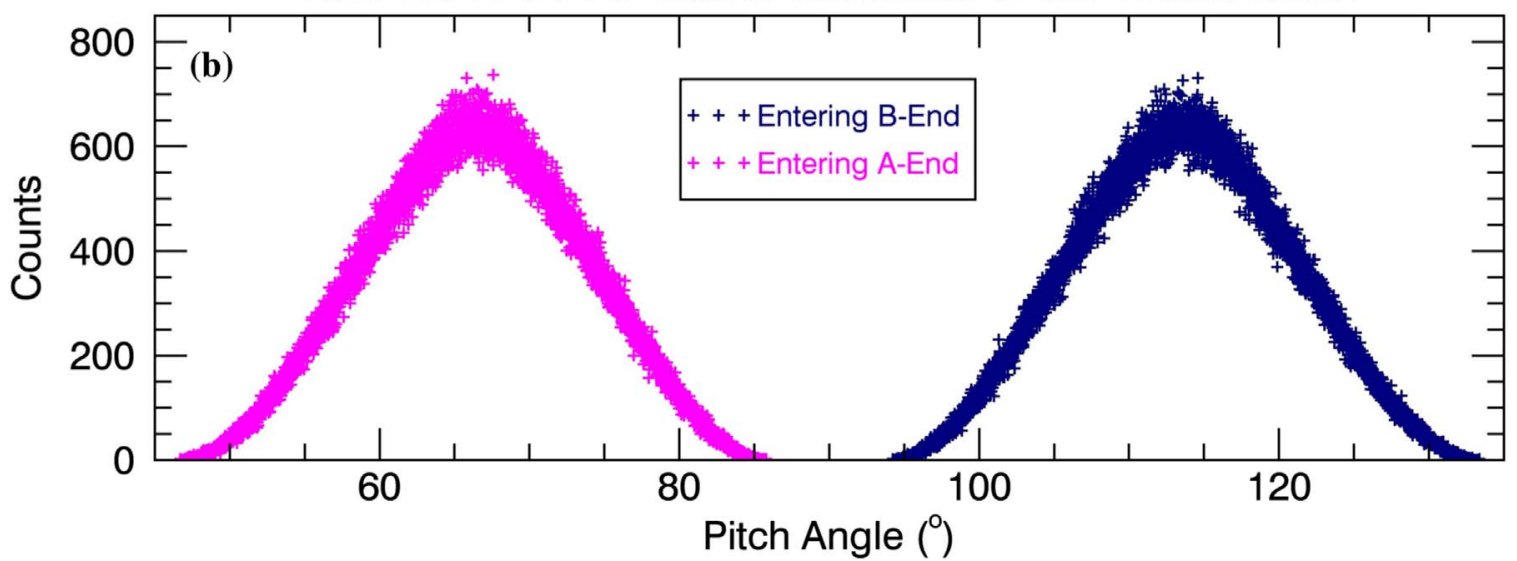

Figure 18. Similar to Figure 17, but for HET 2. (a) HET 2's observed rate reduction was $1.7 \% \pm 0.4 \%$ for this series of maneuvers. (b) Because of HET 2's boresight orientation, Model \#1 produces no reduction when a $4^{\circ} 0$-wide notch is applied to this telescope's simulated bidirectional $70^{\circ}$-offset response function. Note that HET 1 and HET 2 are oppositely oriented, so particles entering the HET 2's B-end are on the right (navy blue) and particles entering HET 2's A-end are on the left (pink).

Table 3

A Summary of Effective Notch Widths (Obtained from Bidirectional Roll Maneuver Fits to PENH Rates; $>70 \mathrm{MeV}$, Proton-dominated) and Corresponding Relative Intensity Changes Arising from the Particle Pitch Angle Anisotropy for Simulated and Observed $70^{\circ}$-offset and Omnidirectional Observations for HET 1

\begin{tabular}{|c|c|c|c|c|c|}
\hline $\begin{array}{l}\text { Roll Maneuver } \\
\text { Interval }\end{array}$ & $\begin{array}{l}\text { Effective Notch } \\
\text { Width }\end{array}$ & $\begin{array}{l}70^{\circ} \text {-offset Simulations } \\
\text { (HET 1 PENH) }\end{array}$ & $\begin{array}{l}70^{\circ} \text {-offset Observations } \\
\text { (HET } 1 \text { PENH) }\end{array}$ & $\begin{array}{l}\text { Omnidirectional Simulations } \\
\text { (HET } 1 \text { Guards) }\end{array}$ & $\begin{array}{c}\text { Omnidirectional Observations } \\
\text { (HET } 1 \text { Guards) }\end{array}$ \\
\hline $2012-263$ & $2.5 \pm 0.4$ & $0.991 \pm 0.002$ & NA & $0.978 \pm 0.003$ & $0.995 \pm 0.002$ \\
\hline $2012-307$ & $2.6 \pm 0.5$ & $0.959 \pm 0.007$ & $0.973 \pm 0.004$ & $0.977 \pm 0.004$ & $0.989 \pm 0.005$ \\
\hline $2013-71$ & $2.0 \pm 0.4$ & $0.963 \pm 0.007$ & $0.957 \pm 0.004$ & $0.982 \pm 0.003$ & $0.984 \pm 0.001$ \\
\hline $2013-122$ & $3.7 \pm 0.4$ & $0.925 \pm 0.008$ & $0.939 \pm 0.004$ & $0.967 \pm 0.003$ & $0.976 \pm 0.001$ \\
\hline $2013-214$ & $0.9 \pm 0.4$ & $0.983 \pm 0.008$ & $0.991 \pm 0.004$ & $0.992 \pm 0.003$ & $0.996 \pm 0.001$ \\
\hline $2014-30$ & $0.3 \pm 0.4$ & $0.995 \pm 0.006$ & $0.994 \pm 0.004$ & $0.998 \pm 0.003$ & $0.998 \pm 0.001$ \\
\hline $2014-69$ & $0.3 \pm 0.4$ & $0.992 \pm 0.009$ & NA & $0.997 \pm 0.003$ & $0.995 \pm 0.001$ \\
\hline 2014-121 & $1.2 \pm 0.4$ & $0.976 \pm 0.008$ & $0.989 \pm 0.004$ & $0.989 \pm 0.003$ & $1.006 \pm 0.001$ \\
\hline $2014-213$ & $1.6 \pm 0.4$ & $0.959 \pm 0.010$ & NA & $0.986 \pm 0.003$ & $0.993 \pm 0.001$ \\
\hline $2014-260$ & $0.2 \pm 0.3$ & $0.997 \pm 0.004$ & $1.012 \pm 0.004$ & $0.998 \pm 0.002$ & $0.998 \pm 0.001$ \\
\hline 2014-304 & $0.8 \pm 0.5$ & $0.987 \pm 0.009$ & $1.007 \pm 0.005$ & $0.993 \pm 0.004$ & $0.994 \pm 0.001$ \\
\hline $2015-310$ & $4.0 \pm 0.4$ & $0.878 \pm 0.012$ & $0.890 \pm 0.003$ & $0.965 \pm 0.003$ & $0.967 \pm 0.001$ \\
\hline $2016-35$ & $3.8 \pm 0.6$ & $0.909 \pm 0.013$ & $0.886 \pm 0.004$ & $0.967 \pm 0.005$ & $0.981 \pm 0.001$ \\
\hline
\end{tabular}


Table 3

(Continued)

\begin{tabular}{lccccc}
\hline \hline $\begin{array}{l}\text { Roll Maneuver } \\
\text { Interval }\end{array}$ & $\begin{array}{c}\text { Effective Notch } \\
\text { Width }\end{array}$ & $\begin{array}{c}70^{\circ} \text {-offset Simulations } \\
\text { (HET 1 PENH) }\end{array}$ & $\begin{array}{c}70^{\circ} \text {-offset Observations } \\
\text { (HET 1 PENH) }\end{array}$ & $\begin{array}{c}\text { Omnidirectional Simulations } \\
\text { (HET 1 Guards) }\end{array}$ & $\begin{array}{c}\text { Omnidirectional Observations } \\
\text { (HET 1 Guards) }\end{array}$ \\
\hline $2016-84$ & $2.3 \pm 0.5$ & $0.960 \pm 0.008$ & NA & $0.980 \pm 0.004$ & $0.986 \pm 0.001$ \\
$2016-126$ & $1.8 \pm 0.5$ & $0.996 \pm 0.001$ & $0.967 \pm 0.004$ & $0.985 \pm 0.004$ & $0.985 \pm 0.001$ \\
$2016-218$ & $1.6 \pm 0.4$ & $0.995 \pm 0.001$ & $0.951 \pm 0.004$ & $0.986 \pm 0.003$ & $0.989 \pm 0.001$ \\
$2016-309$ & $0.7 \pm 0.4$ & $0.998 \pm 0.001$ & $0.982 \pm 0.009$ & $0.994 \pm 0.003$ & $0.989 \pm 0.001$ \\
\hline
\end{tabular}

Note. The time periods shown in italics indicate intervals during which the anisotropy is most prominent, superimposed in Figure 2. Simulated intensities are normalized to values obtained from notch-free simulated response functions. Observed $70^{\circ}$-offset intensities are normalized to temporally adjacent non offset rates, and omnidirectional observations are normalized to the average values during the 2013.6 to 2014.1 time period when count rates are relatively uniform and isotropic. Data are plotted in Figure 4 .

Table 4

Similar to Table 3, but for HET 2 (Plotted in Figure 5)

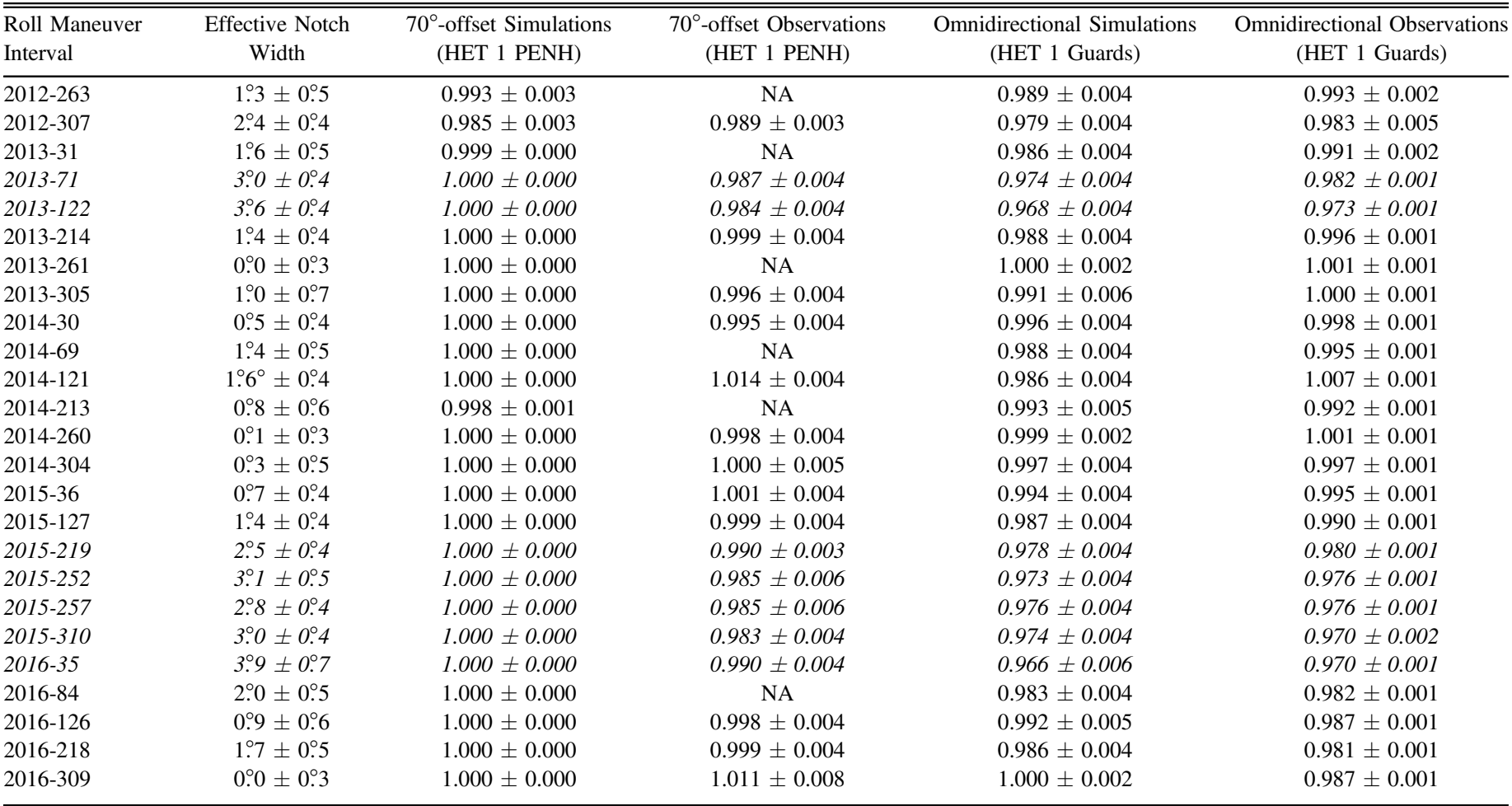

repeat steps 1-7 to accumulate the desired number of particles.

\section{A.4. Model \#1 Results}

Here, we summarize Model \#1's results in tabular form. Table 3 lists the effective notch widths, simulated and observed $70^{\circ}$-offset intensity reductions, and simulated and observed omnidirectional intensity reductions-all for HET 1 (also plotted in Figure 4). Table 4 lists the same quantities for HET 2 (plotted in Figure 5).

\section{Appendix B \\ Model \#2: Partially Filled Notch \\ B.1. Omnidirectional Response Function}

Model \#2 utilizes a two-parameter representation of the notch by introducing a depth term to allow for the possibility of scattering. We achieve this in the omnidirectional notch response function by modifying $j_{n}$ in Equation (6) to allow

\section{"Partially-Filled Notch"}

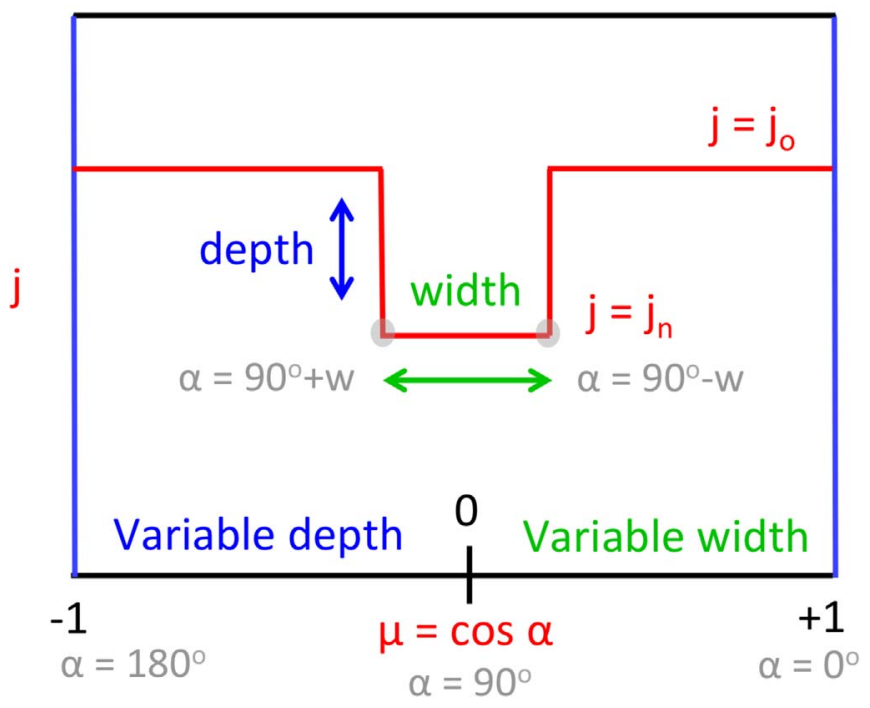

Figure 19. Diagram of notch model \#2. 
Table 5

Summary of HET 1 and HET 2 Observational Values Used for $70^{\circ}$-offset and Omnidirectional Simulations

\begin{tabular}{|c|c|c|c|c|c|c|}
\hline Offset Interval & $2013-67$ & $2013-120$ & $2015-208$ & $2015-250$ & $2015-296$ & 2016-31 \\
\hline Maneuver Days & $67,68,69,70,71$ & $\begin{array}{c}120,121 \\
122\end{array}$ & $\begin{array}{l}208,209 \\
210,215 \\
216\end{array}$ & $250,251,252$ & $\begin{array}{l}296,297,298, \\
300,301,302, \\
303,307,308, \\
312\end{array}$ & $\begin{array}{c}31,32,34,38 \\
39,40\end{array}$ \\
\hline HET 1 Offset Boresight (A-end) & $\begin{aligned} R & =-0.494 \\
T & =-0.675 \\
N & =-0.548\end{aligned}$ & $\begin{array}{l}-0.495 \\
-0.673 \\
-0.550\end{array}$ & $\begin{array}{l}-0.503 \\
-0.669 \\
-0.547\end{array}$ & $\begin{array}{l}-0.506 \\
-0.669 \\
-0.545\end{array}$ & $\begin{array}{l}-0.505 \\
-0.671 \\
-0.543\end{array}$ & $\begin{array}{l}-0.496 \\
-0.678 \\
-0.543\end{array}$ \\
\hline HET 1 Boresight Pitch Angle & $\alpha=78.5$ & 79.3 & 77.2 & 76.7 & 81.3 & 78.3 \\
\hline HET 2 Offset Boresight (B-end) & $\begin{array}{c}R=-0.209 \\
T=-0.056 \\
N=0.976\end{array}$ & $\begin{array}{c}-0.212 \\
-0.056 \\
0.976\end{array}$ & $\begin{array}{l}-0.210 \\
-0.051 \\
0.976\end{array}$ & $\begin{array}{c}-0.207 \\
-0.051 \\
0.977\end{array}$ & $\begin{array}{c}-0.204 \\
-0.050 \\
0.978\end{array}$ & $\begin{array}{c}-0.206 \\
-0.050 \\
0.977\end{array}$ \\
\hline HET 2 Boresight Pitch Angle & $\alpha=69^{\circ} .2$ & 70.0 & 66.1 & 67.1 & 66.2 & $67^{\circ} .2$ \\
\hline $70^{\circ}$-offset Reduction $\left(\delta_{70^{\circ}}\right)$ & $\begin{array}{l}\text { HET } 1=4.3 \pm 0.4 \% \\
\text { HET } 2=1.3 \pm 0.4 \%\end{array}$ & $\begin{array}{l}6.1 \pm 0.4 \% \\
1.6 \pm 0.4 \%\end{array}$ & $\begin{array}{l}7.1 \pm 0.4 \% \\
1.0 \pm 0.4 \%\end{array}$ & $\begin{array}{l}7.2 \pm 0.6 \% \\
1.5 \pm 0.6 \%\end{array}$ & $\begin{array}{c}11.0 \pm 0.3 \% \\
1.7 \pm 0.4 \%\end{array}$ & $\begin{array}{l}11.4 \pm 0.4 \% \\
1.0 \pm 0.4 \%\end{array}$ \\
\hline Omnidirectional Reduction $\left(\delta_{\text {omni }}\right)$ & $\begin{array}{l}\text { HET } 1=1.6 \pm 0.05 \% \\
\text { HET } 2=1.8 \pm 0.05 \%\end{array}$ & $\begin{array}{l}2.4 \pm 0.05 \% \\
2.7 \pm 0.05 \%\end{array}$ & $\begin{array}{l}1.9 \pm 0.05 \% \\
2.1 \pm 0.05 \%\end{array}$ & $\begin{array}{l}2.2 \pm 0.07 \% \\
2.4 \pm 0.07 \%\end{array}$ & $\begin{array}{l}2.9 \pm 0.04 \% \\
3.1 \pm 0.05 \%\end{array}$ & $\begin{array}{l}3.1 \pm 0.06 \% \\
3.3 \pm 0.06 \%\end{array}$ \\
\hline$B$-field (nT) & $\begin{array}{c}R=0.175 \\
T=-0.444 \\
N=0.200 \\
|B|=0.517\end{array}$ & $\begin{array}{c}0.178 \\
-0.421 \\
0.188 \\
0.495\end{array}$ & $\begin{array}{c}0.118 \\
-0.402 \\
0.197 \\
0.463\end{array}$ & $\begin{array}{c}0.117 \\
-0.392 \\
0.183 \\
0.448\end{array}$ & $\begin{array}{c}0.152 \\
-0.379 \\
0.200 \\
0.455\end{array}$ & $\begin{array}{c}0.132 \\
-0.370 \\
0.180 \\
0.433\end{array}$ \\
\hline
\end{tabular}




\section{Omnidirectional \& 70'-offset Response Function Curves: 2013-120 (HET 1)}

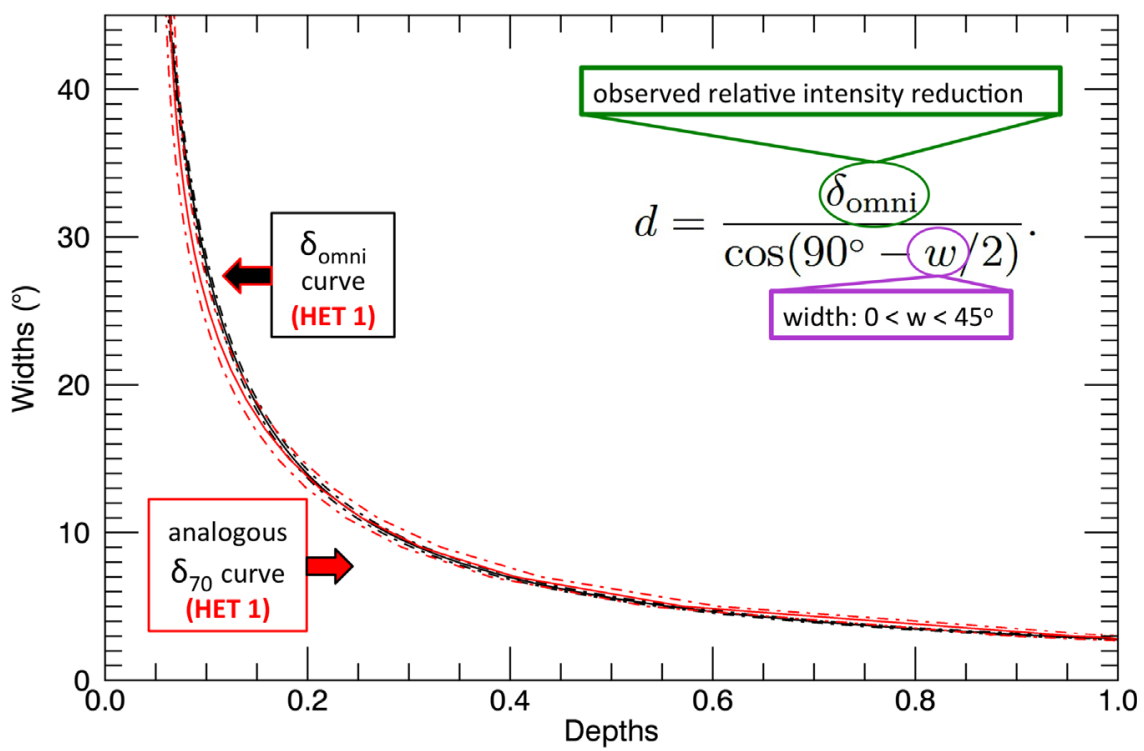

Figure 20. Simulated omnidirectional (black, solid) and $70^{\circ}$-offset (solid, red) widths vs. depths for HET 1 during the 2013-120 offset. The dotted curves reflect the $1 \sigma$ uncertainties in the omnidirectional (black) and $70^{\circ}$-offset (red) measurements.

\section{Omnidirectional \& 70'-offset Response Function Curves: 2013-120 (HET 2)}

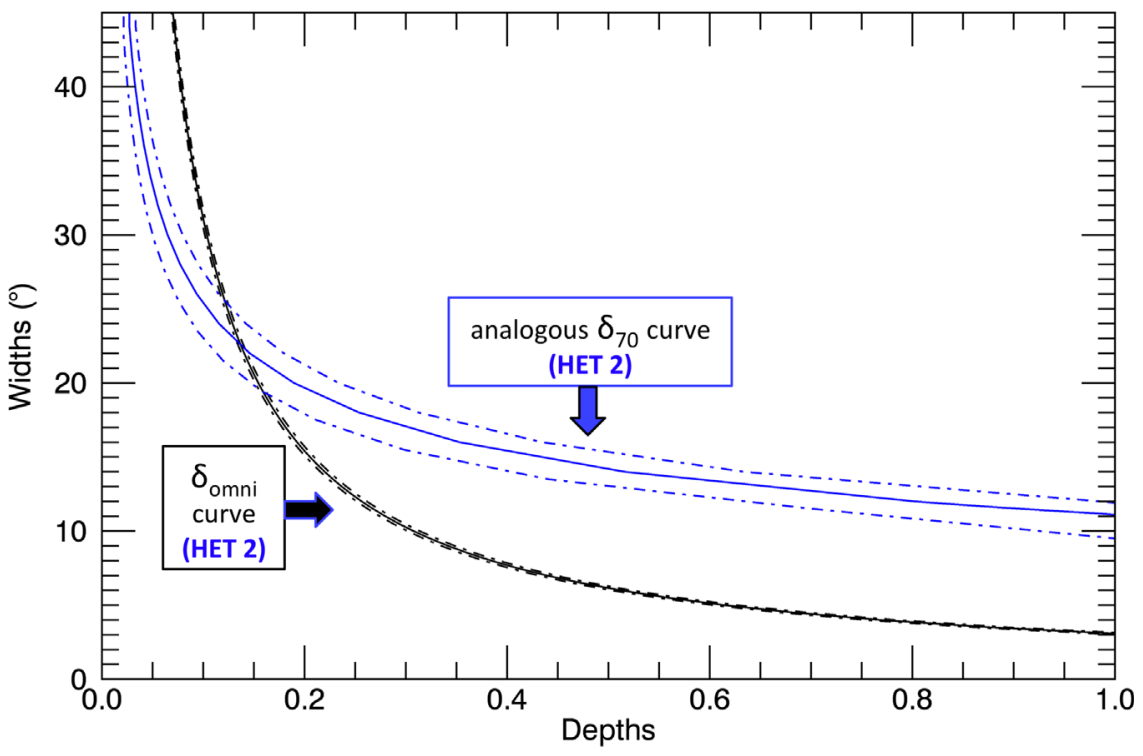

Figure 21. HET 2 version of Figure 20.

for a reduced directional intensity representation of the missing particle distribution $\left(j_{n}<j_{0}\right)$, leading to:

$$
\delta_{\mathrm{omni}}=\frac{J_{n}}{J}=\frac{j_{n}}{j_{0}} \cos \left(90^{\circ}-w / 2\right)=d \times \mu .
$$

Hence, the notch is now partially filled and characterized by an "effective area" of depth, $d=\frac{j_{n}}{j_{0}}$ and width $\mu=\cos \alpha$ ranging from $\alpha=90^{\circ}+w / 2$ to $\alpha=90^{\circ}-w / 2$, as shown in Figure 19.

For a given period where the anisotropy is prominent $\left(\delta_{\text {omni }}>0\right)$, the range of possible widths is no larger than LECP's full telescope opening angle: $0^{\circ} \leqslant w \leqslant 45^{\circ}$.
Therefore, the range of possible depths is given by:

$$
d=\frac{\delta_{\text {omni }}}{\cos \left(90^{\circ}-w / 2\right)} \text {. }
$$

\section{B.2. Directional Response Functions}

Because the anisotropy is now represented as a single function with two unknowns-width and depth-we employ alternate strategies to implement and evaluate the effectiveness of Model \#2's simulations. One strategy is to extend the roll maneuver fits to allow for two parameters-variable width and depth. We thereby acquire independent best-fit notch geometries for HET 1 and HET 2 and compare their results. For example, the 120-122 roll maneuver is characterized by a 


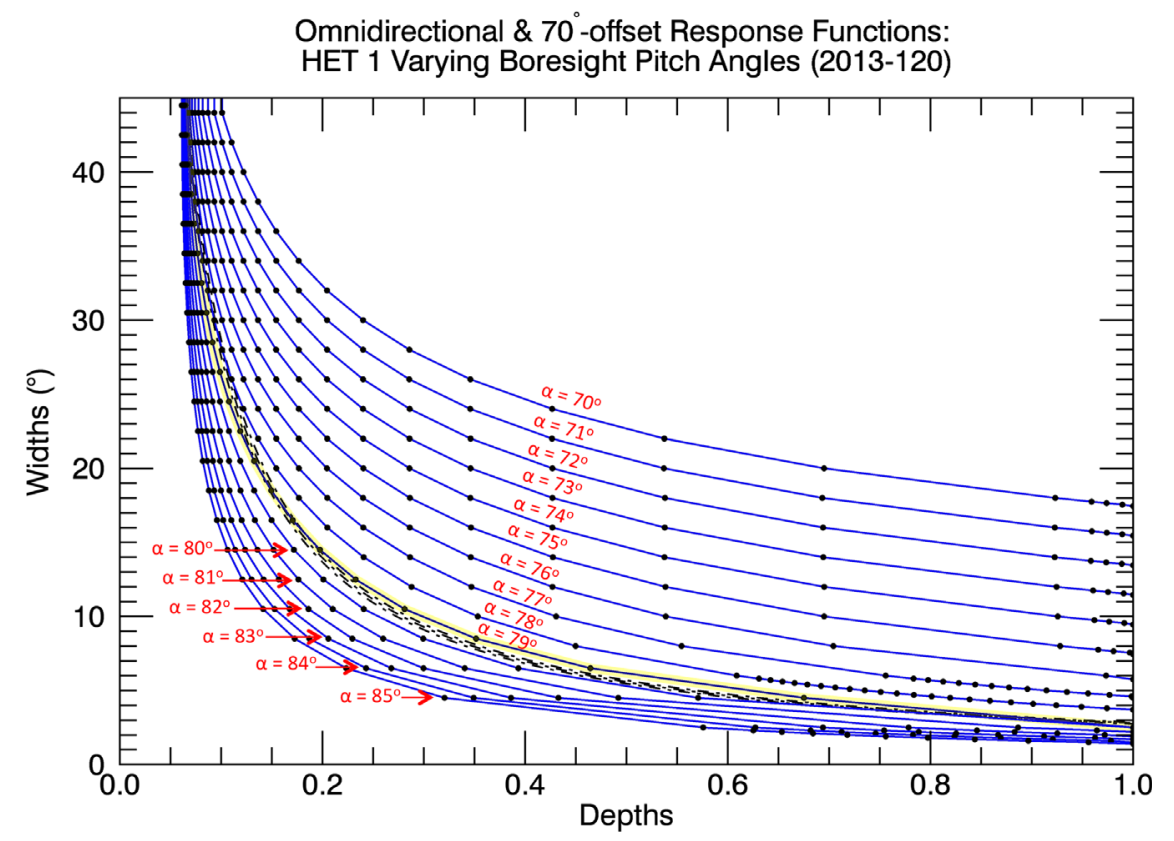

Figure 22. Width and depth curves for simulated HET $170^{\circ}$-offset (blue, solid), and omnidirectional (black, dotted) notch response functions for pitch angles ranging from $\alpha=70^{\circ}$ to $85^{\circ}$. These pitch angles reflect the angle between the telescope's B-end boresight with respect to the magnetic field. The $70^{\circ}$-offset curves were each calculated from observations listed in Table 5 (uncertainties not shown). HET 1's boresight pitch angle during the 2013-120 sequence of 70 ${ }^{\circ}$-offsets was $\alpha=79^{\circ} .3$ (yellow).

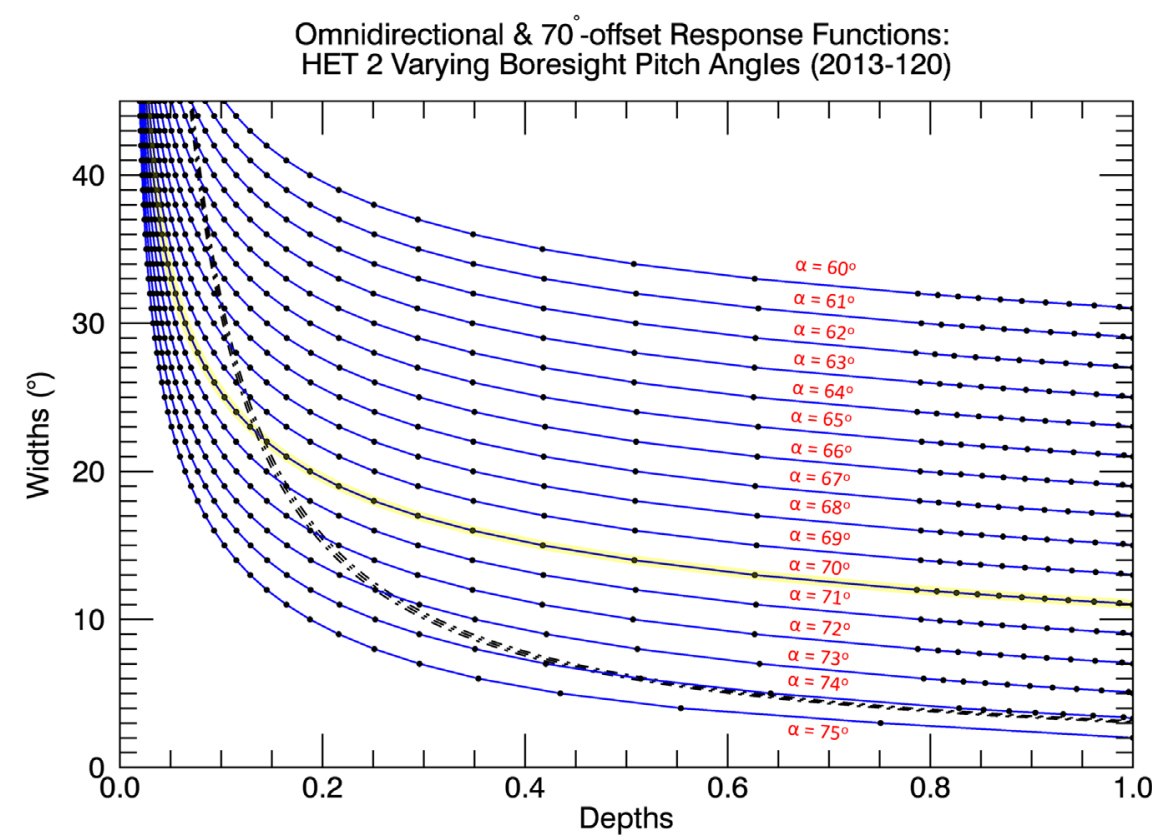

Figure 23. Width and depth curves for simulated HET $270^{\circ}$-offset (blue, solid) and omnidirectional (black, dotted) notch response functions, for pitch angles ranging from $\alpha=60^{\circ}$ to $75^{\circ}$. The pitch angles shown are with respect to HET 2's A-end boresight; its nominal $70^{\circ}$-offset boresight pitch angle during the 2013-120 sequence was $\alpha=70^{\circ} 0$ (yellow).

nominal width of $25^{\circ} .8$ (ranging from $23^{\circ} .2$ to $33^{\circ} .4$ ) and depth of $18.5 \%$ (ranging from $21.5 \%$ to $15.5 \%$ ) for HET 1 . For HET 2, the nominal width and depth is $24^{\circ} .6$ and $18.0 \%$ (ranging from 20.3 to $29^{\circ} .2$ and $21.0 \%$ to $15.0 \%$, respectively).

A second strategy is to determine widths and depths that achieve consistency between each telescope's omnidirectional and $70^{\circ}$-offset response functions, as detailed in the following subsection. The assumption here is that omnidirectional and directional rates are responding to the same notch geometry, and the expectation is that response function curves should differ enough to set at least some limits on the notch's widths and depths.

\section{B.2.1. Omnidirectional and $70^{\circ}$-offset Response Function Curves}

Because of the time-varying nature of the anisotropy and a weak intensity reduction observed by HET 2 during its $70^{\circ}$ offsets, the analysis for Model \#2 focuses on the six offset 
Omnidirectional \& $70^{\circ}$-offset Response Function Curves: 2016-31 (HET 1)

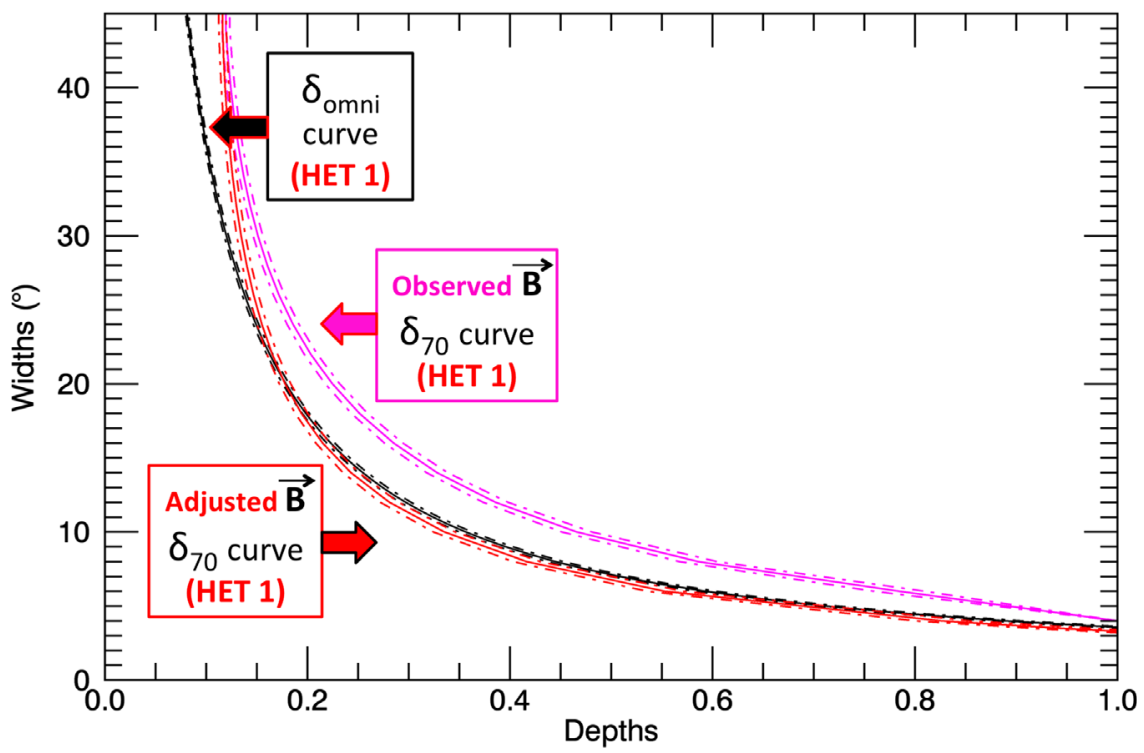

Figure 24. Simulated HET 1 omnidirectional (black, dotted) and two $70^{\circ}$-offset response function curves indicating the difference between the observed $B$-field (pink) of $(0.132,-0.370,0.180) \mathrm{nT}$ (in $R, T, N)$ and an adjusted $B$-field (red) of $(0.181,-0.351,0.170) \mathrm{nT}$ during the 2016-31 offset. HET 1 's B-end $70^{\circ}$-offset boresight pitch angle was $79^{\circ} .3$ for the observed case and 82.5 for the adjusted case.

Partially-Filled Notch Geometries:
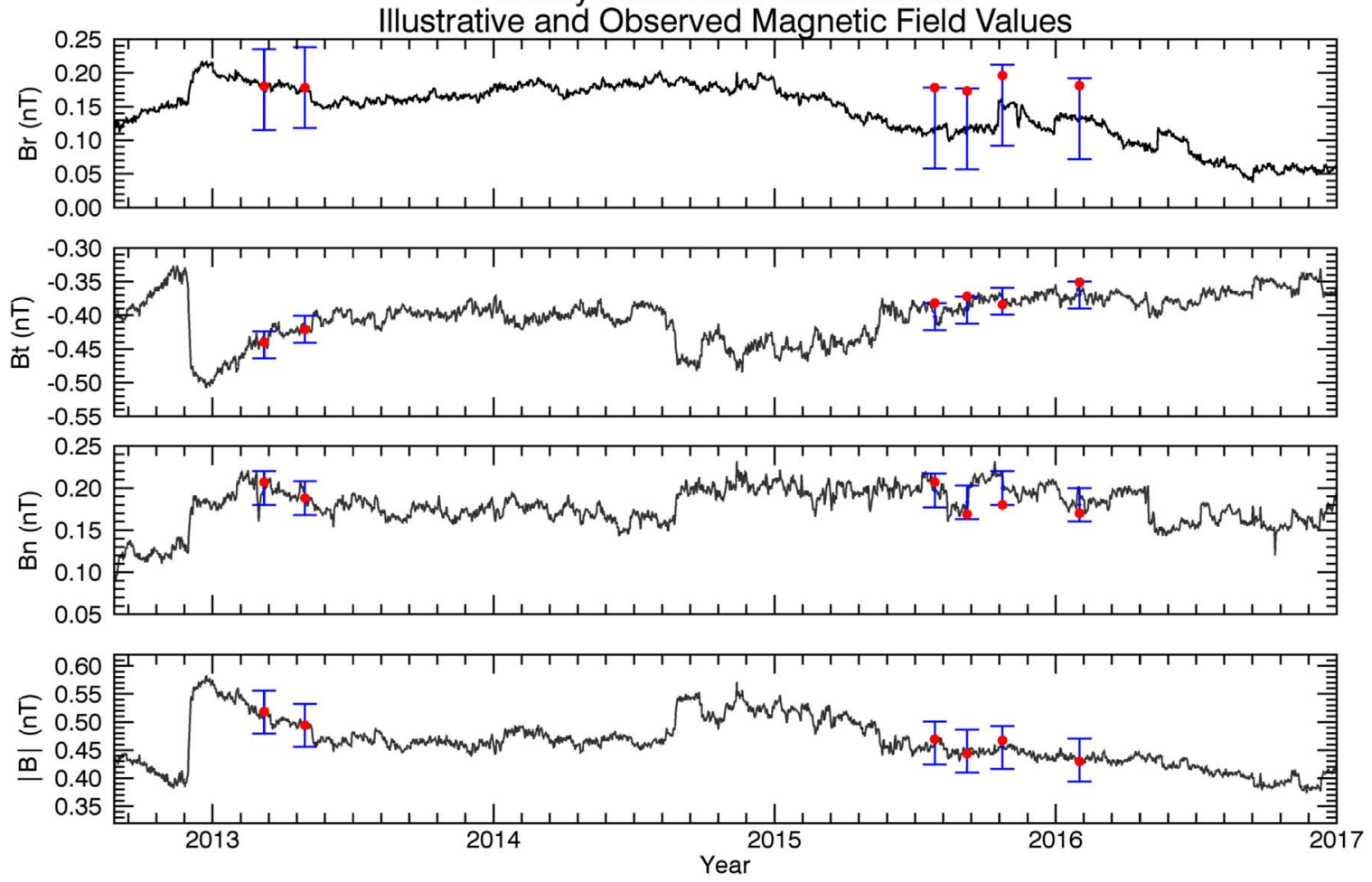

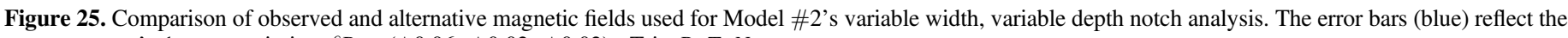
magnetometer's $1 \sigma$ uncertainties: $\delta B=( \pm 0.06, \pm 0.02, \pm 0.02) \mathrm{nT}$ in $R, T, N$.

intervals where the anisotropy is most prominent, listed in Table 5. The telescope orientations, omnidirectional intensity reductions $\left(\delta_{\text {omni }}\right), 70^{\circ}$-offset reductions $\left(\delta_{70^{\circ}}\right)$, and magnetic field observations all reflect the average taken over the offset maneuver sequence time periods.

From the observed omnidirectional intensity reduction $\left(\delta_{\text {omni }}\right)$, we determine the combination of widths and depths that produce results consistent with the observations and uncertainties using widths $\left(0^{\circ} \leqslant w \leqslant 45^{\circ}\right)$ and depths informed by Equation (9). We take a similar approach to determine the $70^{\circ}$-offset isocontours, using the observed relative intensity reduction $\left(\delta_{70^{\circ}}\right)$ and a given telescope's simulated response function to numerically determine depth (analogous to solving Equation (9)). 
Table 6

Summary of Observed and Predicted Magnetic Fields Used for the Variable Width, Variable Depth Notch Analysis

\begin{tabular}{|c|c|c|c|c|c|c|c|}
\hline & & $2013-67$ & $2013-120$ & $2015-208$ & $2015-250$ & $2015-296$ & $2016-31$ \\
\hline \multirow[t]{3}{*}{ Observed Magnetic Field (nT) } & $B r=$ & 0.175 & 0.178 & 0.118 & 0.117 & 0.152 & 0.132 \\
\hline & $B t=$ & -0.444 & -0.421 & -0.402 & -0.392 & -0.379 & -0.370 \\
\hline & $B n=$ & 0.200 & 0.188 & 0.197 & 0.183 & 0.200 & 0.180 \\
\hline \multirow[t]{2}{*}{ Alternative Magnetic Field (nT) } & $B r=$ & 0.180 & 0.178 & 0.178 & 0.173 & 0.196 & 0.181 \\
\hline & $B t=$ & -0.440 & -0.421 & -0.382 & -0.372 & -0.384 & -0.351 \\
\hline \multirow[t]{3}{*}{$\Delta B(\mathrm{nT})$} & $\Delta B r=$ & 0.005 & 0.000 & 0.060 & 0.056 & 0.044 & 0.049 \\
\hline & $\Delta B t=$ & 0.004 & 0.000 & 0.020 & 0.020 & -0.005 & 0.019 \\
\hline & $\Delta B n=$ & 0.007 & 0.000 & 0.010 & -0.014 & -0.020 & -0.010 \\
\hline
\end{tabular}

Note. $\Delta B$ represents the difference between the alternative and observed magnetic fields. The magnetometer's $1 \sigma$ uncertainties are $\delta B=( \pm 0.06, \pm 0.02, \pm 0.02) \mathrm{nT}$ nT in $R, T, N$.

Table 7

HET 1 Roll Maneuver Fits for Notches of Variable Width and Depth for the Six Intervals of Model \#2

\begin{tabular}{|c|c|c|c|c|c|c|c|}
\hline HET 1: Interval & Nominal Width & Lower Limit & Upper Limit & Nominal Depth & Upper Limit & Lower Limit & $P$-value of $\chi^{2}$ Fit \\
\hline 2013-71 & 26.8 & $19{ }^{\circ} 0$ & $35^{\circ} 9$ & $9.4 \%$ & $12.4 \%$ & $6.4 \%$ & $62.5 \%$ \\
\hline $2013-122$ & 25.8 & 23.2 & 33.4 & $18.5 \%$ & $21.5 \%$ & $15.5 \%$ & $48.7 \%$ \\
\hline $2015-219$ & 28.8 & 22.5 & 34.9 & $12.3 \%$ & $16.3 \%$ & $9.3 \%$ & $94.9 \%$ \\
\hline $2015-310$ & 20.8 & 17.9 & $25^{\circ} 0$ & $22.4 \%$ & $27.4 \%$ & $18.4 \%$ & $18.5 \%$ \\
\hline 2016-35 & 13.4 & 10.6 & 16.4 & $29.5 \%$ & $36.5 \%$ & $23.5 \%$ & $21.8 \%$ \\
\hline
\end{tabular}

Note. $P$-values for all intervals are $>5 \%$, indicative of good $\chi^{2}$ fits. Results are plotted in Figures 8 and 9.

Table 8

Similar to Table 7, but for HET 2

\begin{tabular}{|c|c|c|c|c|c|c|c|}
\hline HET 2: Interval & Nominal Width & Lower Limit & Upper Limit & Nominal Depth & Upper Limit & Lower Limit & $P$-value of $\chi^{2}$ Fit \\
\hline 2013-71 & 34.3 & 29.1 & 42.3 & $12.6 \%$ & $16.6 \%$ & $9.6 \%$ & $56.6 \%$ \\
\hline $2013-122$ & 24.6 & 20.3 & $29^{\circ} .2$ & $18.0 \%$ & $21.0 \%$ & $15.0 \%$ & $70.0 \%$ \\
\hline $2015-219$ & $17^{\circ} 6$ & $13: 2$ & 22.6 & $15.1 \%$ & $19.1 \%$ & $11.1 \%$ & $14.0 \%$ \\
\hline $2015-310$ & 20.8 & 17.1 & 28.8 & $16.1 \%$ & $19.1 \%$ & $13.1 \%$ & $30.9 \%$ \\
\hline 2016-35 & 15.2 & 11.7 & 18.9 & $26.6 \%$ & $33.6 \%$ & $20.6 \%$ & $70.7 \%$ \\
\hline
\end{tabular}

Note. The $p$-values for five of six intervals are $>5 \%$, indicative of good $\chi^{2}$ fits. The exception occurs during the 2015-252 interval, which is proximate to a plasma oscillation that began on $\sim 2015-247$. Results are plotted in Figure 8 .

Table 9

HET 1 Range of Widths and Depths from Intersection of Omnidirectional and $70^{\circ}$-offset Response Function Curves for the Six Intervals where the Anisotropy is Most Prominent

\begin{tabular}{lll}
\hline \hline HET 1: Interval & Range of Widths & Range of Depths \\
\hline $2013-67$ & 2.1 to $>45^{\circ}$ & $100 \%$ to $<4.1 \%$ \\
$2013-120$ & 2.8 to $>45^{\circ}$ & $100 \%$ to $<6.5 \%$ \\
$2015-208$ & 11.1 to $26^{\circ} .5$ & $18.6 \%$ to $8.3 \%$ \\
$2015-250$ & 2.4 to $33^{\circ} .7$ & $100 \%$ to $7.7 \%$ \\
$2015-296$ & 3.3 to $20^{\circ} .6$ & $100 \%$ to $16.5 \%$ \\
$2016-31$ & 13.5 to $25^{\circ} .0$ & $26.0 \%$ to $14.6 \%$ \\
\hline
\end{tabular}

Note. The simulations incorporated values listed in Table 5 and pitch angles determined by the alternative $B$-fields in Table 6 . These results are plotted in Figure 8.
Figures 20 and 21 show superimposed omnidirectional and $70^{\circ}$-offset curves for the 2013-120 offset. While HET 1's observations allow for a broad range of widths and depths2.8 to $>45^{\circ}$ and $100 \%$ to $<6.5 \%$ (Figure 20) - HET 2's observations narrow the range of possible values to widths of $19^{\circ} .2$ to $25^{\circ} .8$ and respective depths of $16.4 \%$ down to $11.8 \%$ along the curve (Figure 21). The 2013-120 nominal values are 22.5 wide and $13.7 \%$ deep.

Differences in HET 1 and 2's boresight orientations enable HET 2 to better set limits to the notch's parameters than HET 1. Indeed, this is true for all offsets. HET 2 is most sensitive to the notch's width and depth because the anisotropy is at the very edge of its field of view. In contrast, the anisotropy is more fully within HET 1's field of view, so it is much more sensitive to the magnetic field direction than HET 2. 
Table 10

HET 2 Nominal Widths and Depths (with Ranges) from Intersection of Omnidirectional and $70^{\circ}$-offset Response Function Curves Incorporating Values Listed in Table 5 and Pitch Angles Determined by the Alternative $B$-fields in Table 6

\begin{tabular}{|c|c|c|c|c|c|c|}
\hline HET 2: Interval & Nominal Width & Lower Limit & Upper Limit & Nominal Depth & Upper Limit & Lower Limit \\
\hline $2013-67$ & 29.2 & 24.2 & 34.3 & $7.0 \%$ & $8.6 \%$ & $5.8 \%$ \\
\hline $2013-120$ & 22.5 & 19.2 & 25.8 & $13.7 \%$ & $16.4 \%$ & $11.8 \%$ \\
\hline $2015-208$ & 28.1 & 23.6 & 32.2 & $8.7 \%$ & $10.6 \%$ & $7.4 \%$ \\
\hline $2015-250$ & 20.4 & 14.4 & 26.2 & $13.4 \%$ & $19.5 \%$ & $10.2 \%$ \\
\hline $2015-296$ & 18.1 & $15^{\circ} 4$ & 20.8 & $19.8 \%$ & $23.6 \%$ & $17.0 \%$ \\
\hline $2016-31$ & 14.3 & 10.5 & 17.6 & $26.3 \%$ & $36.2 \%$ & $20.9 \%$ \\
\hline
\end{tabular}

Note. These results are plotted Figures 8 and 9 .

To illustrate each telescope's differing sensitivities, HET 1's width versus depth curves for boresight pitch angles ranging from $\alpha=70^{\circ}$ to $85^{\circ}$ are presented for the 2013-120 offset in Figure 22. Similar curves are also shown for HET 2, in this case for boresight pitch angles ranging from $\alpha=60^{\circ}$ to $75^{\circ}$ (Figure 23). Notably, each of HET 2's simulated $70^{\circ}$-offset curves (solid blue) intersect with the omnidirectional curves (dashed black) at some point, revealing a variety of possible solutions, depending on the particular value of $\alpha$. However, the majority of HET 1's curves do not intersect; the few that do represent a narrow range of pitch angles, with curves overlapping so well that the range of possible widths and depths is not effectively constrained by HET 1 alone.

\section{B.3. Model \#2's Alternative Magnetic Fields}

A complication arises because the combination of the telescope's boresight direction and the observed magnetic field direction for most intervals produces disagreement between HET 1's omnidirectional and $70^{\circ}$-offset notch response function curves. In fact, the 2013-120 is the only interval where omnidirectional and $70^{\circ}$-offset simulations intersect without adjustment (albeit, only a small adjustment is needed for 2013-67). For example, Figure 24 shows HET 1's curves for 2016-31. For this interval, there is no strong agreement between the two curves to within their respective uncertainties that also yields a width and depth consistent with HET 2 observations.

Ultimately, the typical shift in boresight pitch angle required to resolve HET 1's disagreement $\left(\sim 3^{\circ} .5\right.$ in $\left.\alpha\right)$ is larger than CRS's expected telescope alignment uncertainties $\left(\lesssim 1^{\circ}\right)$. However, agreement between HET 1 and HET 2 can be achieved using a $B$-field that falls within the magnetometer's $1 \sigma$ uncertainties, $\delta B=( \pm 0.06, \pm 0.02, \pm 0.02) \mathrm{nT}$ in $R, T, N$. Thus, we perform an additional search for alternate $B$-field directions that achieve agreement among HET 1 and 2 omnidirectional and directional observations for each interval. The results of this search are listed in Table 6 and shown in Figure 25. In principle, differing combinations of $B_{r}, B_{t}$, and $B_{n}$ can produce identical pitch angles for HET 1 , so other solutions could exist. Nonetheless, we select each component by minimizing its deviation from the reported measurement.

\section{B.4. Model \#2 Results}

Tables 7 and 8 list the fit results for independently calculated roll maneuvers for HET 1 and HET 2, allowing for notches of variable width and depth. Tables 9 and 10 list the notch parameters obtained using $70^{\circ}$-offset and omnidirectional response function curves for HET 1 and HET 2, respectively.

\section{ORCID iDs}

J. S. Rankin (1) https://orcid.org/0000-0002-8111-1444

A. C. Cummings (1) https://orcid.org/0000-0002-3840-7696

D. J. McComas 주 https://orcid.org/0000-0001-6160-1158

\section{References}

Burlaga, L. F., Florinski, V., \& Ness, N. F. 2015, ApJL, 804, L31

Burlaga, L. F., Florinski, V., \& Ness, N. F. 2018, ApJ, 854, 20

Burlaga, L. F., \& Ness, N. F. 2016, ApJ, 829, 134

Burlaga, L. F., Ness, N. F., Gurnett, D. A., \& Kurth, W. S. 2013, ApJL, 778, L3

Cummings, A. C., Stone, E. C., Heikkila, B. C., et al. 2016, ApJ, 831, 18

Decker, R. B., Krimigis, S. M., Roelof, E. C., \& Hill, M. E. 2012, Natur, 489, 124

Funsten, H. O., Allegrini, F., Crew, G. B., et al. 2009, Sci, 326, 964

Funsten, H. O., DeMajistre, R., Frisch, P. C., et al. 2013, ApJ, 776, 30

Gurnett, D. A., Kurth, W. S., Allendorf, S. C., \& Poynter, R. L. 1993, Sci, 262, 199

Gurnett, D. A., Kurth, W. S., Burlaga, L. F., \& Ness, N. F. 2013, Sci, 341,1489

Gurnett, D. A., Kurth, W. S., Stone, E. C., et al. 2015, ApJ, 809, 121

Jokipii, J. R., \& Kóta, J. 2014, ApJL, 794, L4

Kóta, J., \& Jokipii, J. R. 2017, ApJ, 839, 126

Krimigis, S. M., Decker, B., Roelof, E., et al. 2013, Sci, 341, 144

McComas, D. J., Allegrini, F., Bochsler, P., et al. 2009a, SSRv, 146, 11

McComas, D. J., Allegrini, F., Bochsler, P., et al. 2009b, Sci, 326, 959

McComas, D. J., \& Schwadron, N. A. 2014, ApJL, 795, L17

Pogorelov, N. V., Heerikhuisen, J., Roytershteyn, V., et al. 2017, ApJ, 845, 9

Pogorelov, N. V., Heerikhuisen, J., Zank, G. P., et al. 2011, ApJ, 742, 104

Ptuskin, V. S. 2001, SSRv, 99, 281

Richardson, J. D., Wang, C., Liu, Y. D., et al. 2017, ApJ, 834, 190

Schwadron, N. A., Bzowski, M., Crew, G. B., et al. 2009, Sci, 326, 966

Schwadron, N. A., Moebius, E., Fuselier, S. A., et al. 2014, ApJS, 215, 13

Stone, E. C., Cummings, A. C., McDonald, F. B., et al. 2013, Sci, 341, 150

Stone, E. C., Vogt, R. E., McDonald, F. B., et al. 1977, SSRv, 21, 355

Washimi, H., Tanaka, T., \& Zank, G. P. 2017, ApJL, 846, L9

Washimi, H., Zank, G. P., \& Hu, Q. 2011, MNRAS, 416, 1475

Webber, W. R., \& McDonald, F. B. 2013, GeoRL, 40, 1665

Zirnstein, E. J., Heerikhuisen, J., Funsten, H. O., et al. 2016, ApJL, 818, L18 